\title{
LES FLUX HYDRO-SÉDIMENTAIRES DANS LE BASSIN SUPÉRIEUR DU FLEUVE LOIRE (MASSIF CENTRAL, FRANCE) AU COURS DES TROIS DERNIERS MILLÉNAIRES : ARCHIVES SÉQUENTIELLES, CHRONOLOGIE ET CORRÉLATIONS RÉGIONALES
}

\author{
Emmanuelle DEFIVE ${ }^{1,2}$, Jean-François BERGER ${ }^{3}$, Alexandre POIRAUD ${ }^{1,2}$, \\ Adrien BARRA ${ }^{3}$, Emma BOUVARD $^{4}$, Clément VIRMOUX ${ }^{5}$, Olivier VOLDOIRE ${ }^{1,2}$, \\ Alexandre GARREAU ${ }^{1,2}$, Yannick MIRAS ${ }^{1,2}$, Aude BEAUGER ${ }^{1,2}$, \\ Manon CABANIS ${ }^{1,2}$, Yanni GUNNELL ${ }^{3}$, Régis BRAUCHER ${ }^{6}$, \\ André-Marie DENDIEVEL ${ }^{7}$, Sébastien NOMADE ${ }^{8}$, Vincent DELVIGNE ${ }^{9}$, \\ Audrey LAFARGE ${ }^{10}$, René LIABEUF ${ }^{11}$, Hervé GUILLOU ${ }^{8} \&$ Jean-Paul RAYNAL ${ }^{12}$
}

\section{RÉSUMÉ}

Une recherche paléoenvironnementale et géoarchéologique pluridisciplinaire a été conduite dans le bassin supérieur du fleuve Loire en vue d'apprécier, à partir d'une chronostratioraphie multi-proxy, la variabilité des flux hydro-sédimentaires dans les têtes de bassin du réseau hydrographique à l'échelle de l'Holocène. Sur la base de 107 datations par le radiocarbone obtenues sur l'ensemble du secteur étudié, une analyse statistique par densité de probabilité a permis de dégager une périodisation de l'évolution du bassin versant à l'échelle des trois derniers millénaires. Celle-ci concorde avec les connaissances déjà acquises sur l'axe voisin de la moyenne vallée du Rhône. Quatre crises érosives majeures se manifestent au début de La Tène (vers 400 av. J.-C.), à la transition entre la fin de l'âge du Fer et le Haut-Empire romain (100 av. J.-C.-100 ap. J.-C.), dans la seconde partie du Bas-Empire romain et au début du haut Moyen Âge (350-550 ap. J.-C.) puis entre le XIV et la fin du XIX ${ }^{\mathrm{e}}$ siècle. La tripartition classique du PAG est également vérifiée dans cet environnement de moyenne montagne, mais ici enrichie par la mise en évidence d'une période inédite de stabilisation temporaire des hydrosystèmes élémentaires au XVIII ${ }^{\mathrm{e}}$ siècle. Entre ces crises principales, le haut Moyen Âge apparaît comme une période d'instabilité environnementale, tandis qu'une longue période de grande stabilité se développe entre la fin du IX siècle et le début du XIII ${ }^{\mathrm{e}}$ siècle, en coïncidence avec le Petit Optimum Médiéval.

Mots-clés : flux hydro-sédimentaires, radiocarbone, densité de probabilité cumulée (CPDF), Loire amont, Holocène récent, Petit Âge Glaciaire

\footnotetext{
${ }^{1}$ Université Clermont Auvergne, Université Blaise Pascal, GEOLAB, BP 10448, FR-63000 CLERMONT-FERRAND.

Courriel : emmanuelle.defive@univ-bpclermont.fr ; apoiraud@yahoo.fr ; olivier.voldoire@univ-bpclermont.fr ;

alexandre.garreau@univ-bpclermont.fr ; yannick.miras@univ-bpclermont.fr ; aude.beauger@univ-bpclermont.fr ; manon.cabanis@inrap.fr

${ }^{2}$ CNRS, UMR 6042, GEOLAB, FR-63057 CLERMONT-FERRAND.

${ }^{3}$ Univ. Lyon Lumière, UMR CNRS 5600 EVS, 5 avenue Pierre Mendès-France, FR-69676 BRON Cedex.

Courriel : jean-francois.berger@univ-lyon2.fr ; adrien.barra@live.fr ; yanni.gunnell@univ-lyon2.fr

${ }^{4}$ UMR 5138 CNRS, Archéométrie et Archéologie, MSH Maison de 1'Orient et de la Méditerranée - Jean Pouilloux, 7 rue Raulin, FR-69365

LYON Cedex 7. Courriel : emma bouvard@yahoo.fr

${ }^{5}$ UMR 8591 CNRS, Laboratoire de Géographie Physique, 1 place Aristide Briand, FR-92195 MEUDON.

Courriel : clement.virmoux@1gp.cnrs.fr

${ }^{6}$ LN2C-ASTER, CEREGE, UMR 7330 CNRS et Aix-Marseille Universités, Plateau d'Arbois, BP 80, FR-13545 AIX-EN-PROVENCE

Cedex 04. Courriel : braucher@cerege.fr

${ }^{7}$ Univ. Lyon, Université Jean Monnet, UMR CNRS 5600 EVS-ISTHME, 6 rue Basse des Rives, FR-42023 SAINT-ÉTIENNE Cedex 02.

Courriel : andremarie.dendievel@gmail.com

${ }^{8}$ LSCE/IPSL, CEA-CNRS-UVSQ, LSCE-Vallée, Bât. 12, avenue de la Terrasse, FR-91198 GIF-SUR-YVETTE Cedex.

Courriel : sebastien.nomade@1sce.ipsl.fr

${ }^{9}$ Service de Préhistoire, Université de Liège, Place du 20 août, 7 (Bât. A1), BE-4000 Liège, et PACEA, De la Préhistoire à 1'Actuel, Cultures, Environnement, Anthropologie, université de Bordeaux, Bât. B8, allée Geoffroy Saint-Hilaire, CS 50023, FR-33615 PESSAC Cedex.

Courriel : vincent.delvigne@hotmail.fr

${ }^{10}$ UMR 5140 CNRS, Archéologie des sociétés méditerranéennes et Université de Montpellier, 390 avenue de Pérols, FR-34970 LATTES.

Courriel : mouf331@gmail.com

${ }^{11}$ SRA, DRAC Auvergne-Rhône-Alpes, Hôtel de Chazerat, 4 rue Blaise Pascal, FR-63010 CLERMONT-FERRAND Cedex 1.

Courriel : rene.liabeuf@culture.gouv.fr

${ }^{12}$ UMR 5199 CNRS - PACEA, De la Préhistoire à l'Actuel, Cultures, Environnement, Anthropologie, Bât. B8, allée Geoffroy Saint-Hilaire, CS 50023, FR-33615 PESSAC Cedex et Max Planck Institute for Evolutionary Anthropology, Department of Human Evolution, Deutscher

Platz 6, 04103 Leipzig, Germany. Courriel :.jpraynal@wanadoo.fr
} 
THE THREE LAST MILLENIA HISTORY OF THE HOLOCENE HYDRO-SEDIMENTARY FLOWS IN THE UPSTREAM LOIRE RIVER BASIN (MASSIF CENTRAL, FRANCE): CONTRIBUTION OF A CUMULATIVE PROBABILITY DENSITY FUNCTION (CPDF) ANALYSIS AND REGIONAL CORRELATIONS

A paleoenvironnemental and geoarchelogical pluridisciplinary and multi-proxy research was carried out on the upstream Loire basin to study the hydro-sedimentary flows wich have affected headwaters of the hydrosystem during the Holocene. A set of 107 radicarbon dates support the results. A statistical analysis (Cumulative Probability Density Function) defined the different evolution stages during the last three millenia. The results are similar to those obtained in the the Rhone Valley. Four major erosional crisis occurred circa the beginning of second Iron Age (around $400 \mathrm{BC}$ ), the end of Iron Age and the beginning of the Roman period (100 BC-100 AD), in the Late Antiquity and at the beginning of the Upper Middle Age (350-550 AD) and finally between the XIV ${ }^{\text {th }}$ and the end of the XIX ${ }^{\text {th }}$ century. The classic subdivision of the LIA in three phases is here enriched by a new period of hydrosystems stability during the XVIII ${ }^{\text {th }}$ century. Between these major crises, the Early Middle Age appears as an unstable environmental period, while a long period of stability occurred between the end of the $\mathrm{IX}^{\text {th }}$ century and the beginning of the XIII ${ }^{\text {th }}$ century, during the medieval little climatic optimum.

Keywords: hydro-sedimentary flows, radiocarbon, cumulative probability density function (CPDF), Loire upstream, Late Holocene, Little Ice Age

\section{1 - INTRODUCTION}

L'analyse des populations d'âges ${ }^{14} \mathrm{C}$ par densité de probabilité cumulée (ou CPDF : cumulative probability density function) est une méthode statistique d'analyse des fréquences de distribution de valeurs quantitatives descriptives d'un phénomène. Cette méthode s'est fortement développée depuis la fin des années 1990 dans les domaines de la paléohydrologie continentale et de l'archéologie (Macklin \& Lewin, 2003 ; Magny, 2004 ; Michczynska \& Pazdur, 2004). Les géographes spécialistes des milieux fluviaux l'ont adoptée et généralisée aux bassins-versants européens les mieux documentés dans le domaine de la paléodynamique fluviale (Johnstone et al., 2006 ; Starkel et al., 2006 ; Berger et al., 2008 ; Hoffmann et al., 2008a,b ; Zielhofer \& Faust, 2008 ; Macklin et al., 2010 ; Benito et al., 2015 ; Carozza et al., 2015). L'approche par CPDF des phases de paléopédogenèse et de turfigenèse des plaines alluviales reste, quant à elle, encore peu documentée au niveau mondial (Berger et al., 2008 ; Hofmann et al., 2008a,b ; Zielhofer et al., 2010 ; Berger, 2015). Une analyse chronostratigraphique des phases de développement des tourbières d'altitude existe parfois à l'échelle régionale (en France : Forez, Velay ; Cubizolle et al., 2007, 2012), mais les processus en jeu (alimentation en eau, anthropisation) sont différents des processus qui gouvernent les alternances entre sédimentation terrigène et accumulation organique dans un environnement fluvial. En outre, ce type d'analyse a encore été peu appliqué aux têtes de bassin des réseaux hydrographiques, qui se localisent fréquemment en région de moyenne montagne. En France, ces domaines ont été quelque peu délaissés par la recherche sur le fonctionnement des hydrosystèmes et il est donc rare d'y disposer d'un ensemble de datations suffisant pour contraindre la réponse des versants et des cours d'eau aux forçages environnementaux. Le Massif central, où les études sur l'histoire environnementale et morpho-paysagère des têtes de réseau hydrographique sont encore peu nombreuses, ne fait pas exception à ce constat (Ballut, 2001 ; Allée, 2003 ; Cubizolle, 2005 ; Gob, 2005 ; Schmitt et al., 2005 ; Ballut \& Trément, 2006 ; Jacob et al., 2006 ; Miras et al., 2006, 2014 ; Ballut et al., 2008 ; Gob et al., 2008 ; Larue, 2009 ;
Cubizolle et al., 2012, 2016 ; Lespez, 2012 ; L'Héritier, 2012 ; Jacob \& Astrade, 2014 ; Delile et al., 2016).

Nous présentons ici les résultats de l'application de la méthode CPDF aux observations litho-stratigraphiques effectuées dans le bassin supérieur de la Loire où se développent depuis peu des études paléoenvironnementales et géoarchéologiques visant à reconstituer l'histoire des flux hydro-sédimentaires dans les cours d'eau élémentaires des têtes de réseau et à mesurer l'impact environnemental du climat et des sociétés à l'échelle de l'Holocène au moins (programmes WRACC-Liger et AGES).

\section{2 - TERRAIN D'ÉTUDE ET MÉTHODOLOGIE}

\section{1 - LE BASSIN SUPÉRIEUR DE LA LOIRE}

Le bassin supérieur de la Loire se développe sur les hautes terres cristallines volcanisées de la bordure sudorientale du Massif central (Velay - Vivarais) distribuées entre 700 et $1500 \mathrm{~m}$ et culminant au mont Mézenc à $1754 \mathrm{~m}$, au carrefour d'influences climatiques atlantiques, montagnardes (vent, neige, gel) et méditerranéennes (averses cévenoles) (Valadas, 1984 ; Mergoil \& Boivin, 1993 ; Defive, 1996 ; Defive et al., 2011 ; Defive \& Poiraud, 2013) (fig. 1). Il est constitué d'un dense réseau de vallons élémentaires et de petites vallées (écoulements d'ordre Strahler 3 à 4 et inférieur ; Strahler, 1957) dont les dépôts colluviaux et alluviaux révèlent, à l'échelle de l'Holocène au moins, une histoire des flux hydro-sédimentaires riche en événements de fréquence et d'intensité variable. La stratigraphie des petites plaines alluviales reflète une succession d'épisodes de stabilité et d'instabilité relative dans les bassins versants, qui correspondent en première hypothèse à des alternances de périodes de stockage et de déstockage dans la cascade sédimentaire. Dans cette région de hautes terres comme ailleurs, les fluctuations environnementales enregistrées par les séquences stratigraphiques étudiées reflètent néanmoins une réponse parfois équivoque $\mathrm{du}$ système aux variations du climat et aux modalités de l'occupation humaine (Defive, 2010, 2013 ; Defive et al., 2011, 2015). 


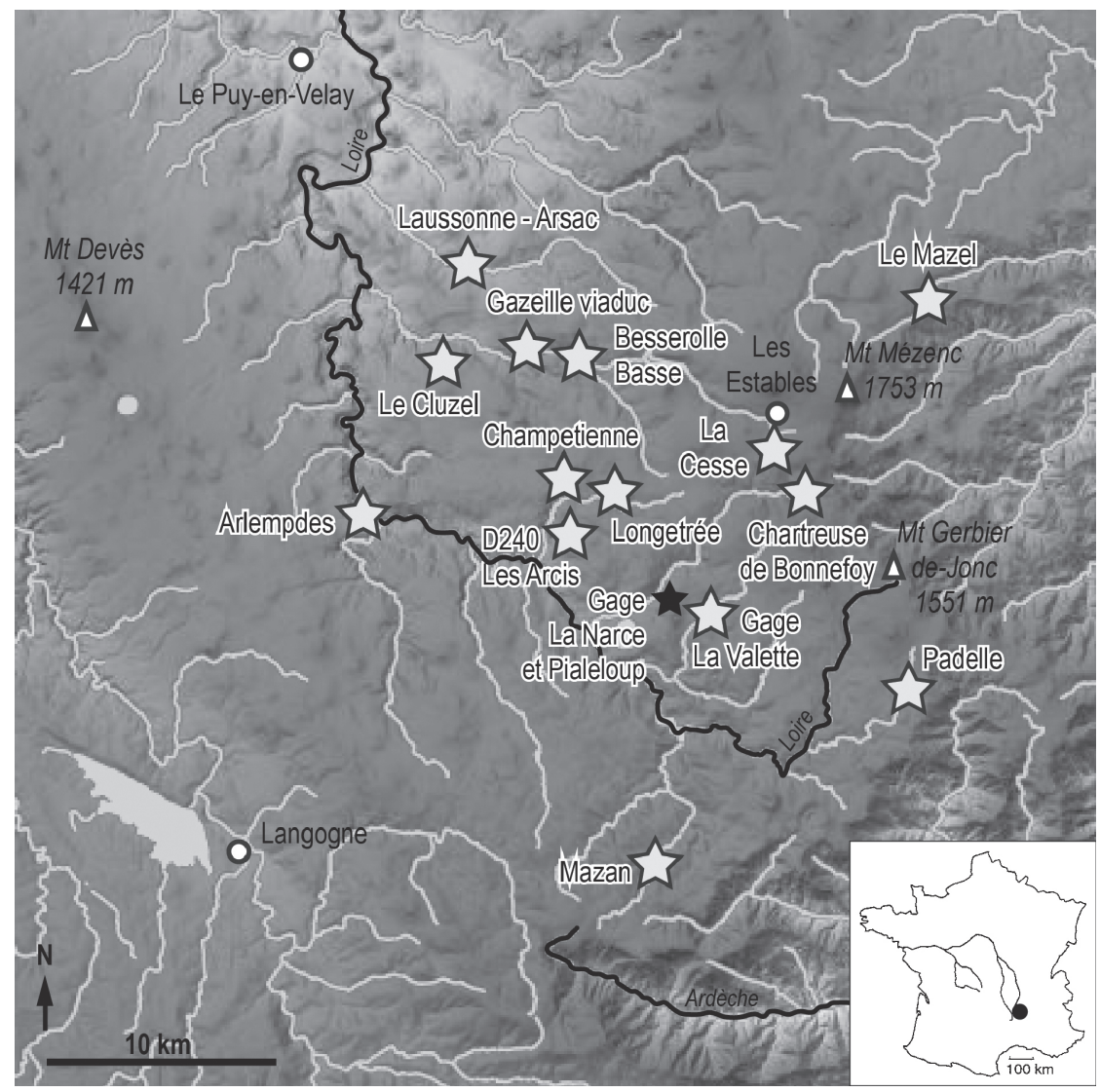

Fig. 1 : Carte oro-hydrographique du bassin supérieur de la Loire (Velay-Vivarais, sud-est du Massif central)

Étoiles blanches : sites datés $\left({ }^{14} \mathrm{C}\right)$ utilisés pour l'analyse CPDF en vue du phasage de l'évolution hydro-pédo-sédimentaire à l'échelle des trois derniers millénaires. Étoile noire : site de La Narce et Pialeloup étudié par Dendievel et al. (2015).

Fig. 1: Oro-hydrographic map of the upper Loire catchment (Velay-Vivarais, SE of Massif Central). White stars indicate dated sites (14C) used for the CPDF analysis. Black star indicate the site of La Narce and Pialeloup studied by Dendievel et al. (2015).

\section{2 - UNE RECHERCHE PLURIDISCIPLINAIRE ET MULTI-PROXY}

\subsection{1 - Une étude multi-proxy des remblaiements de} fond de vallon

La recherche s'est concentrée sur l'étude des remblaiements de fond de vallon observés dans les coupes naturelles de berge et dans plusieurs tranchées ouvertes en travers des vallons. Sur le terrain, l'étude litho-stratigraphique des dépôts a été complétée par un relevé topographique précis (DGPS et station totale) et par la réalisation de profils géo-électriques visant à détecter le contact socle / dépôts et à évaluer l'épaisseur des remplissages. L'échantillonnage a concerné l'ensemble du haut bassin de la Loire et plusieurs coupes ont été privilégiées pour une étude à haute résolution (bassin versant de Champetienne, vallées du Gage et de l'Orcival) (fig. 1 et 2). Les échantillons ont donné lieu à une analyse multi-proxy destinée à déterminer les caractéristiques dynamiques et environnementales de mise en place des dépôts : sédimentologie, susceptibilité magnétique, géochimie (XRF, core-scanner), palynologie, carpologie, macro-restes, diatomées. Enfin, des études d'archives et des investigations de terrain ont permis de recenser et de cartographier les traces d'occupation humaine, de mise en valeur et d'aménagement (bâti, murets, ouvrages liés à la gestion de l'eau etc.) susceptibles, au même titre que le mode d'occupation du sol et les pratiques culturales, d'avoir eu un impact sur les flux hydro-sédimentaires. La présente étude se focalise sur l'âge des séquences organo-détritiques observées, et sur leur signification en termes de forçages paléoclimatiques et de changements d'utilisation du sol.

\subsection{2 - Datation par ${ }^{14} \mathrm{C}$ des séquences litho-strati- graphiques et analyse CPDF des données obtenues}

Au total, 107 datations ont été effectuées sur 24 sites (tab. 1), principalement par la méthode du radiocarbone (essentiellement par SMA), pour établir la trame chronologique indispensable aux corrélations entre séquences, à la quantification des flux et à la réflexion sur les facteurs de contrôle des évolutions. L'essentiel des datations a été effectué sur charbons, une petite partie sur des bois non carbonisés, de la tourbe ou du sédiment organique.

L'analyse CPDF repose sur un échantillonnage considéré comme aléatoire - de dates cumulées au sein des formations pédo-sédimentaires d'un bassin-versant. Les bases de données utilisées sont souvent peu critiquées en amont des analyses, mais certaines limites à 1'application de cette méthode ont malgré tout été discutées (Shennan \& Edinborough, 2007 ; Williams, 2012), comme par exemple la taille critique de l'échantillon de dates ${ }^{14} \mathrm{C}$ utilisé. Sur un unique site d'étude stratifié, 


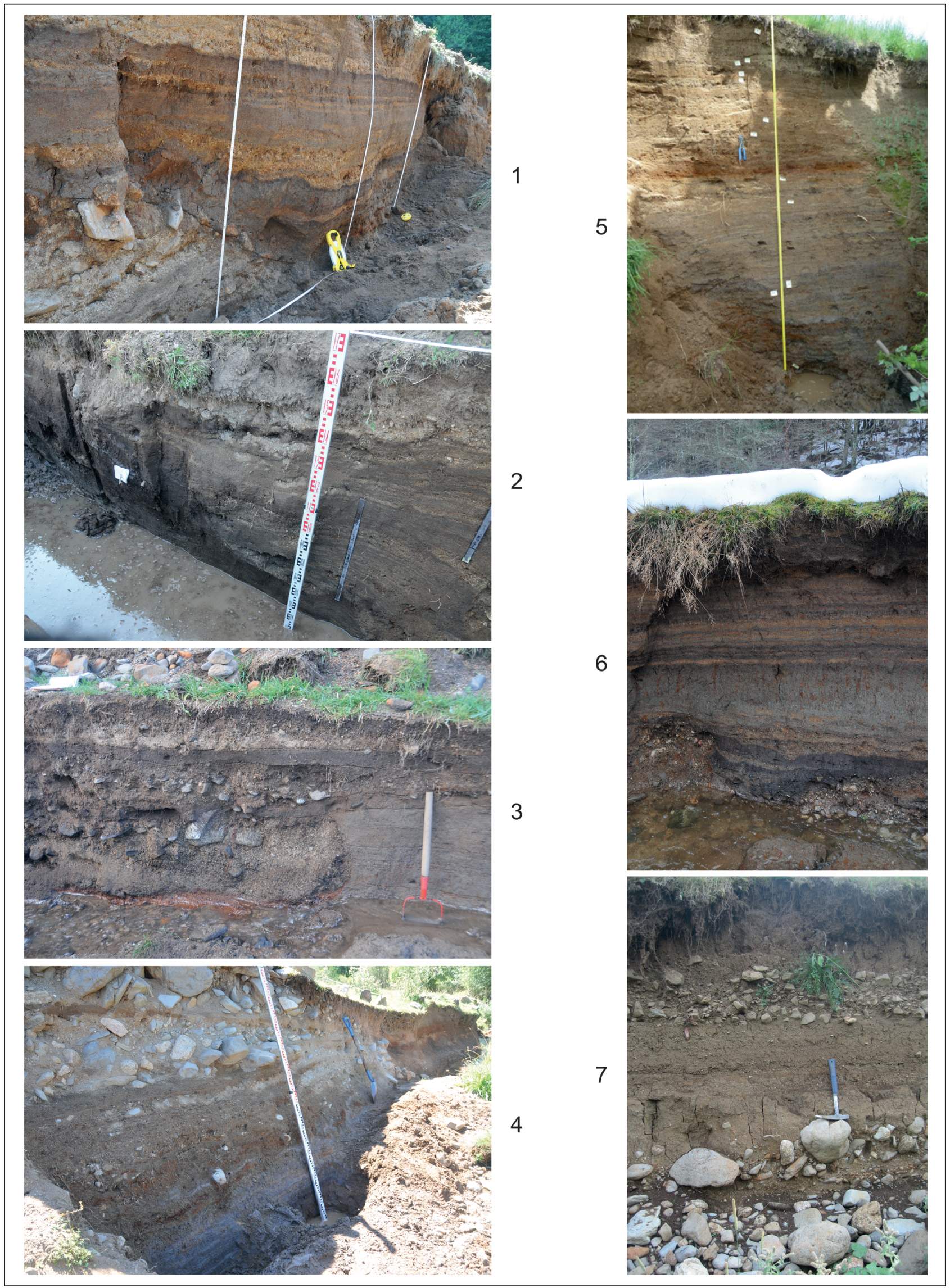

Fig. 2 : Exemples choisis parmi les coupes étudiées (localisation des sites sur la fig. 1).

1/ Vallon de Champetienne, tranchée $2 ; 2$ / Vallon de Champetienne, tranchée $1 ; 3 /$ Tranchée dans le lit moyen de l'Orcival $; 4 /$ Coupe de berge en rive gauche du Gage sous la ferme de La Valette ; 5/ Flanc de ravine au Mazel (commune de Saint-Clément) ; 6/ Vallon de Champetienne, coupe de berge ; 7/ Coupe de berge en rive gauche de la Gazeille en amont du viaduc de la Recoumène.

Fig. 2: Views of some studied sections (sites locations on fig. 1). 1/ Champetienne dale, trench 2; 2/ Champetienne dale, trench 1; 3/ Trench in middle bed of Orcival valley; 4/ La Valette bank (Gage valley); 5/ Gully near Le Mazel (Saint-Clément); 6/ Champetienne dale bank; 7/ Gazeille bank near Recoumène viaduct. 


\begin{tabular}{|c|c|c|c|c|c|c|c|}
\hline Site & Contexte morpho-pédo-sédimentaire & $\begin{array}{l}\text { Code } \\
\text { CPDF }\end{array}$ & Code labo & $\begin{array}{l}\text { Matériel } \\
\text { daté }\end{array}$ & $\begin{array}{l}\text { Âge BP } \\
\text { conv. }\end{array}$ & $\begin{array}{l}\text { Âge BC/AD } \\
\text { cal. 1s }\end{array}$ & $\begin{array}{l}\text { Âge } \mathrm{BC} / \mathrm{AD} \\
\text { cal. } 2 \mathrm{~s}\end{array}$ \\
\hline Gazeille viaduc & CB. Alluvions limono-sableuses à débris végétaux & STA & Beta 341158 & bois & $840 \pm 30$ & $1170-1220 \mathrm{AD}$ & $1059-1264 \mathrm{AD}$ \\
\hline Gazeille viaduc & CB. Alluvions limono-sableuses à débris végétaux & STA & Ly-9837 & bois & $830 \pm 30$ & $1188-1251 \mathrm{AD}$ & 1161-1264 AD \\
\hline Vallon du Cluzel & CB. Alluvions sablo-limoneuses à débris végétaux & STA & Beta 341308 & bois & $280 \pm 30$ & $1530-1540 \mathrm{AD}$ & 1498-1795 AD \\
\hline $\begin{array}{l}\text { Champetienne } \\
\text { Log T1haut }\end{array}$ & $\begin{array}{l}\text { CT. Colluvions granitiques pédogénisées (paléo- } \\
\text { sol) }\end{array}$ & STA & Beta 386220 & charbon & $9110 \pm 30$ & $8300-8285$ BC & $8424-8257 \mathrm{BC}$ \\
\hline $\begin{array}{l}\text { Champetienne } \\
\text { Log T1haut }\end{array}$ & $\begin{array}{l}\text { CT. Colluvions granitiques pédogénisées (paléo- } \\
\text { sol) }\end{array}$ & STA & Beta 386221 & charbon & $4020 \pm 30$ & $2575-2485$ BC & $2620-2471 \mathrm{BC}$ \\
\hline $\begin{array}{l}\text { Champetienne Log } \\
\text { T1haut }\end{array}$ & CT. Paléosol cumulique sur colluvions & STA & Beta 386223 & charbon & $1140 \pm 30$ & $885-965 \mathrm{AD}$ & 777-981 AD \\
\hline $\begin{array}{l}\text { Champetienne Log } \\
\text { T1centre }\end{array}$ & $\begin{array}{l}\text { CT. Alluvions sablo-argileuses pédogénisées } \\
\text { (paléosol) }\end{array}$ & STA & Beta 399782 & charbon & $1970 \pm 30$ & $5-65 \mathrm{AD}$ & $45 \mathrm{BC}-85 \mathrm{AD}$ \\
\hline $\begin{array}{l}\text { Champetienne Log } \\
\text { T1centre }\end{array}$ & $\begin{array}{l}\text { CT. Alluvions sablo-argileuses pédogénisées } \\
\text { (paléosol) }\end{array}$ & STA & Poz-70703 & charbon & $1195 \pm 30$ & $788-875 \mathrm{AD}$ & $720-941 \mathrm{AD}$ \\
\hline $\begin{array}{l}\text { Champetienne } \\
\text { prolong. T1 }\end{array}$ & CB. Tourbe à fragments de bois & STA & $\begin{array}{l}07 \mathrm{~S}-117 \\
\text { (via BRGM) }\end{array}$ & bois & $1150 \pm 40$ & 778-968 AD & 774-978 AD \\
\hline $\begin{array}{l}\text { Champetienne } \\
\text { prolong.T1 }\end{array}$ & CB. Tourbe à fragments de bois & STA & $\begin{array}{l}07 \mathrm{~S}-118 \\
\text { (via BRGM) }\end{array}$ & bois & $430 \pm 40$ & 1429-1485 AD & 1413-1624 AD \\
\hline Champetienne T2 & CT. Alluvions limono-sableuses riches en MO & STA & Beta 386234 & charbon & $930 \pm 30$ & $1035-1155 \mathrm{AD}$ & $1025-1165 \mathrm{AD}$ \\
\hline Champetienne T2 & CT. Alluvions limoneuses riches en MO & STA & Beta 399780 & charbon & $1290 \pm 30$ & 670-765 AD & 664-770 AD \\
\hline Champetienne T2 & $\begin{array}{l}\text { CT. Alluvions organo-détritiques sablo-limono- } \\
\text { argileuses à nombreux petits débris organiques }\end{array}$ & STA & Beta 386217 & charbon & $150 \pm 30$ & $\begin{array}{l}1670- \\
\text { post } 1950 \mathrm{AD}\end{array}$ & $1667-1950 \mathrm{AD}$ \\
\hline Champetienne T2 & CT. Colluvions sablo-limoneuses pédogénisées & STA & Ly-11580 & charbon & $160 \pm 30$ & $1669-1945 \mathrm{AD}$ & 1664- 1914 AD \\
\hline $\begin{array}{l}\text { Champetienne } \\
\text { coupe JG }\end{array}$ & CB. Fluviosol cumulique & STA & Ly-34073 & charbon & $1220 \pm 30$ & 726-870 AD & 692-887 AD \\
\hline Champetienne T3 & CT. Alluvions limoneuses à débris végétaux & STA & Beta 386235 & $\begin{array}{l}\text { Fibres } \\
\text { végétales }\end{array}$ & $460 \pm 30$ & $1430-1445 \mathrm{AD}$ & $1412-1468 \mathrm{AD}$ \\
\hline Champetienne T5 & CT. Tourbe à nombreux fragments de bois & STA & Beta 392861 & bois & $1690 \pm 30$ & $335-395 \mathrm{AD}$ & $256-416 \mathrm{AD}$ \\
\hline Champetienne T5 & CT. Tourbe à nombreux fragments de bois & STA & Beta 392860 & bois & $1580 \pm 30$ & $420-540 \mathrm{AD}$ & $410-546 \mathrm{AD}$ \\
\hline Champetienne T6 & $\begin{array}{l}\text { CT. Colluvio-alluvions sablo-limono-argileuses } \\
\text { pédogénisées (paléosol) }\end{array}$ & STA & Beta 399777 & charbon & $9510 \pm 30$ & $8820-8765$ BC & $9121-8736$ BC \\
\hline Champetienne T6 & $\begin{array}{l}\text { CT. Colluvio-alluvions sablo-limono-argileuses } \\
\text { pédogénisées à charbons (paléosol) }\end{array}$ & STA & Beta 399779 & charbon & $1380 \pm 30$ & 645-660 AD & 606-680 AD \\
\hline Champetienne T6 & $\begin{array}{l}\text { CT. Colluvio-alluvions sablo-limono-argileuses } \\
\text { pédogénisées à charbons (paléosol) }\end{array}$ & STA & Beta 399778 & charbon & $2190 \pm 30$ & $355-285 \mathrm{BC}$ & $361-178 \mathrm{BC}$ \\
\hline $\begin{array}{l}\text { Champetienne } \\
\text { proche T5 }\end{array}$ & $\begin{array}{l}\text { Cr. Formation organo-détritique tourbeuse à débris } \\
\text { de bois et charbons }\end{array}$ & STA & Beta 367799 & bois & $1480 \pm 30$ & $560-610 \mathrm{AD}$ & 538-645 AD \\
\hline $\begin{array}{l}\text { Champetienne } \\
\text { proche T5 }\end{array}$ & $\begin{array}{l}\text { Cr. Alluvions organo-détritiques tourbeuses à } \\
\text { débris de bois et charbons }\end{array}$ & STA & Beta 367798 & bois & $1260 \pm 30$ & 690-780 AD & 669-865 AD \\
\hline Bouteyre (vallon) & CB. Tourbe avec bois & STA & Beta 367795 & bois & $250 \pm 30$ & $1640-1660 \mathrm{AD}$ & $1521-1950 \mathrm{AD}$ \\
\hline Longetrée & CT. Alluvions limono-sableuses organiques & STA & Poz-70708 & charbon & $180 \pm 30$ & $1667-1930 \mathrm{AD}$ & 1652-1917 AD \\
\hline $\begin{array}{l}\text { D.240 aval } \\
\text { Champetienne }\end{array}$ & C. Colluvions pédogénisées sur granite & STA & $\begin{array}{l}\text { 07S-119 } \\
\text { (via BRGM) }\end{array}$ & bois & $380 \pm 40$ & $1448-1620 \mathrm{AD}$ & $1441-1635 \mathrm{AD}$ \\
\hline La Cesse & CB. Alluvions limoneuses avec bois & STA & Beta 341162 & bois & $950 \pm 30$ & $1030-1050 \mathrm{AD}$ & $1024-1155 \mathrm{AD}$ \\
\hline $\begin{array}{l}\text { Chartreuse de Bon- } \\
\text { nefoy }\end{array}$ & CB. Dépôt organique noirâtre à débris de bois & STA & $\begin{array}{l}\text { 07S-121 } \\
(\text { via BRGM) }\end{array}$ & bois & $230 \pm 40$ & 1641-1939 AD & 1521-1921 AD \\
\hline $\begin{array}{l}\text { Chartreuse de Bon- } \\
\text { nefoy }\end{array}$ & CB. Dépôt organique noirâtre à débris de bois & STA & $\begin{array}{l}\text { 07S-126 } \\
\text { (via BRGM) }\end{array}$ & bois & $270 \pm 40$ & $1523-1795 \mathrm{AD}$ & $1486-1941 \mathrm{AD}$ \\
\hline $\begin{array}{l}\text { Chartreuse de Bon- } \\
\text { nefoy }\end{array}$ & CB. Dépôt organique noirâtre à débris de bois & STA & $\begin{array}{l}\text { 07S-127 } \\
\text { (via BRGM) }\end{array}$ & bois & $430 \pm 40$ & $1429-1485 \mathrm{AD}$ & $1413-1624 \mathrm{AD}$ \\
\hline $\begin{array}{l}\text { Chartreuse de Bon- } \\
\text { nefoy }\end{array}$ & CB. Argile sableuse organique à cailloux et blocs & STA & $\begin{array}{l}\text { 07S-124 } \\
\text { (via BRGM) }\end{array}$ & bois & $400 \pm 40$ & $1442-1617$ AD & $1432-1633 \mathrm{AD}$ \\
\hline $\begin{array}{l}\text { Chartreuse de Bon- } \\
\text { nefoy }\end{array}$ & CB. Argile sableuse organique à cailloux et blocs & STA & $\begin{array}{l}07 \mathrm{~S}-123 \\
\text { (via BRGM) }\end{array}$ & bois & $380 \pm 40$ & $1448-1620 \mathrm{AD}$ & $1441-1635 \mathrm{AD}$ \\
\hline Gage La Valette & CB. Alluvions argileuses organiques noirâtres & STA & Beta 399784 & charbon & $1410 \pm 30$ & $620-655 \mathrm{AD}$ & $590-665 \mathrm{AD}$ \\
\hline Gage La Valette & CB. Alluvions organo-détritiques tourbeuses & STA & Beta 392852 & charbon & $730 \pm 30$ & $1265-1285 \mathrm{AD}$ & 1224-1298 AD \\
\hline $\begin{array}{l}\text { Arlempdes } \\
\text { La Garnasse }\end{array}$ & CB. Alluvions limoneuses organiques & STA & Beta 386240 & $\begin{array}{l}\text { Sédiment } \\
\text { organique }\end{array}$ & $790 \pm 30$ & $1220-1265 \mathrm{AD}$ & $1190-1279$ AD \\
\hline $\begin{array}{l}\text { Arlempdes } \\
\text { La Garnasse }\end{array}$ & CB. Alluvions limoneuses organiques & STA & Beta 386239 & $\begin{array}{l}\text { Sédiment } \\
\text { organique }\end{array}$ & $390 \pm 30$ & $1450-1490 \mathrm{AD}$ & 1441-1631 AD \\
\hline Le Mazel & CB. Alluvions limono-argileuses organiques & STA & Ly-34076 & charbon & $990 \pm 30$ & 999-1147 AD & 989-1153 AD \\
\hline Mazan cimetière & $\begin{array}{l}\text { CB. Alluvions sablo-limoneuses pédogénisées } \\
\text { (paléosol) }\end{array}$ & STA & Beta 392865 & charbon & $330 \pm 30$ & $1490-1640 \mathrm{AD}$ & $1477-1643 \mathrm{AD}$ \\
\hline Padelle (riv.) & CB. Alluvions sableuses & $\mathrm{AG}$ & Ly-11190 & charbon & $1190 \pm 30$ & 778-878 AD & $722-945 \mathrm{AD}$ \\
\hline Padelle (riv.) & CB. Alluvions argilo-limono-sableuses & $\mathrm{AG}$ & Ly-11189 & charbon & $1215 \pm 30$ & 769-874 AD & 694-889 AD \\
\hline Gage La Valette & CB. Alluvions limono-sablo-argileuses & $\mathrm{AG}$ & Beta 392858 & charbon & $1050 \pm 30$ & 980-1020 AD & 900-1027 AD \\
\hline
\end{tabular}

Tab. 1 : Dates ${ }^{14} \mathrm{C}$ utilisées pour l'analyse CPDF pour le haut bassin de la Loire (localisation des sites sur fig. 1).

$\mathrm{C} /$ Coupe ; Cr/ Carottage manuel ; CB/ Coupe de berge ; CT/ Coupe en tranchée ; MO/ Matière organique ; STA/ Formations indicatrices de stabilité ; AG/ Formations fines d'aggradation, dynamiques de faible énergie ; TOR/ Formations grossières indicatrices de dynamiques torrentielles.

Tab. 1: Radiocarbon dates of the Loire river headwaters used for the CPDF (sites location on fig. 1). C/ Outcrop ; Cr/ Manual coring ; CB/ Riverbank outcrop ; CT/ Trench outcrop ; MO/Organic matter ; STA/Stability periods formations ; AG/Aggradation fine sediments, low dynamics ; TOR/Coarse alluviums, torrential dynamics. 


\begin{tabular}{|c|c|c|c|c|c|c|c|}
\hline Gage La Valette & CB. Alluvions limono-sablo-argileuses & $\mathrm{AG}$ & $\begin{array}{l}\text { Beta } 394882 \\
+392857\end{array}$ & charbon & $390 \pm 30$ & $1450-1490 \mathrm{AD}$ & 1441-1631 AD \\
\hline Gage La Valette & CB. Alluvions sablo-limoneuses & $\mathrm{AG}$ & Beta 392855 & charbon & $470 \pm 30$ & $1425-1445 \mathrm{AD}$ & $1410-1457 \mathrm{AD}$ \\
\hline Gage La Valette & CB. Alluvions sablo-limoneuses & $\mathrm{AG}$ & Beta 392856 & charbon & $490 \pm 30$ & $1415-1440 \mathrm{AD}$ & 1404-1450 AD \\
\hline Gage La Valette & CB. Alluvions limono-sablo-argileuses & $\mathrm{AG}$ & Beta 392854 & charbon & $520 \pm 30$ & 1410-1430 AD & 1324-1443 AD \\
\hline $\begin{array}{l}\text { Champetienne T1 } \\
\text { Log M6 }\end{array}$ & CT. Alluvions sablo-argileuses gleyifiées & AG & Beta 386231 & charbon & $1600 \pm 30$ & 415-535 AD & $399-539 \mathrm{AD}$ \\
\hline $\begin{array}{l}\text { Champetienne T1 } \\
\text { Log M6 }\end{array}$ & CT. Alluvions sableuses en lentilles dans la tourbe & $\mathrm{AG}$ & Beta 386232 & charbon & $200 \pm 30$ & $1660-1950 \mathrm{AD}$ & $1646-1950 \mathrm{AD}$ \\
\hline $\begin{array}{l}\text { Champetienne T1 } \\
\text { Log M6 }\end{array}$ & CT. Alluvions sablo-argileuses gleyifiées & $\mathrm{AG}$ & Beta 386229 & charbon & $2310 \pm 30$ & $400-380 \mathrm{BC}$ & $411-235 \mathrm{BC}$ \\
\hline $\begin{array}{l}\text { Champetienne } \\
\text { T1centre }\end{array}$ & ilo-sableuses nsem & $\mathrm{AG}$ & Poz-70702 & charbon & $2040 \pm 30$ & $92 \mathrm{~B}$ & $46 \mathrm{AD}$ \\
\hline $\begin{array}{l}\text { Champetienne T1 } \\
\text { Log3-4m }\end{array}$ & CT. Alluvions limono-sableuses riches en MO & $\mathrm{AG}$ & Beta 386225 & charbon & $420 \pm 30$ & $1440-1465 \mathrm{AD}$ & $1427-1618 \mathrm{AD}$ \\
\hline Champetienne $\mathrm{T} 2$ & CT. Alluvions sablo-limoneuses à bois et charbons & $\mathrm{AG}$ & Poz-70705 & bois & $1625 \pm 30$ & $390-530 \mathrm{AD}$ & $353-537 \mathrm{AD}$ \\
\hline $\begin{array}{l}\text { Champetienne } \\
\text { Tranchée } 2\end{array}$ & $\begin{array}{l}\text { CT. Alluvions sablo-limoneuses à débris } \\
\text { organiques }\end{array}$ & AG & Beta 386218 & \begin{tabular}{|l} 
Fibres \\
végétales
\end{tabular} & $230 \pm 30$ & 1650-1795 AD & 1530-1950 AD \\
\hline $\begin{array}{l}\text { Champetienne } \\
\text { Tranchée } 2\end{array}$ & $\begin{array}{l}\text { CT. Alluvions sablo-limoneuses à débris orga- } \\
\text { niques }\end{array}$ & $\mathrm{AG}$ & 6219 & \begin{tabular}{|l|} 
Fibres \\
végétales
\end{tabular} & $340 \pm 30$ & 1485 & $0 \mathrm{AD}$ \\
\hline $\begin{array}{l}\text { Champetienne } \\
\text { Tranchée } 2\end{array}$ & $\begin{array}{l}\text { CT. Alluvions sablo-limoneuses à débris orga- } \\
\text { niques }\end{array}$ & AG & 36216 & $\begin{array}{l}\text { Sédiment } \\
\text { organique }\end{array}$ & $400 \pm 30$ & 1445-1485 AD & $5 \mathrm{AD}$ \\
\hline Champetienne T2 & CT. Alluvions limono-sableuses riches en MO & $\mathrm{AG}$ & Beta 386233 & charbon & $830 \pm 30$ & $1190-1250 \mathrm{AD}$ & $1161-1264 \mathrm{AD}$ \\
\hline $\begin{array}{l}\text { Champetienne } \\
\text { Tranchée } 3\end{array}$ & CT. Colluvions granitiques à bois et charbons & AG & Ly-11581 & $\begin{array}{l}\text { Bois car- } \\
\text { bonisé }\end{array}$ & $1200 \pm 30$ & 775-873 AD & 715-940 AD \\
\hline Champetienne T3 & $\begin{array}{l}\text { CT. Alluvions limono-sableuses à débris orga- } \\
\text { niques }\end{array}$ & $\mathrm{AG}$ & Beta 386236 & charbon & $2530 \pm 30$ & 785-595 BC & $797-543 \mathrm{BC}$ \\
\hline $\begin{array}{l}\text { Champetienne } \\
\text { proche T5 }\end{array}$ & $\begin{array}{l}\text { Cr. Alluvions sablo-argileuses gleyiques à débris } \\
\text { organiques et charbons }\end{array}$ & $\mathrm{AG}$ & 797 & charbon & \pm 30 & $\mathrm{BC}$ & $\mathrm{BC}$ \\
\hline $\begin{array}{l}\text { Champetienne T6 } \\
\log 13-14 \mathrm{~m}\end{array}$ & CT. Alluvions riches en débris de bois et fibres & $\mathrm{AG}$ & Poz-70712 & bois & $1515 \pm 30$ & 474-600 AD & $428-615 \mathrm{AD}$ \\
\hline $\begin{array}{l}\text { Champetienne } \\
\text { coupe JG }\end{array}$ & CB. Alluvions riches en débris de bois & $\mathrm{AG}$ & Ly-34075 & charbon & $1640 \pm 30$ & 353-505 AD & $336-535 \mathrm{AD}$ \\
\hline $\begin{array}{l}\text { Champetienne } \\
\text { coupe JG }\end{array}$ & CB. A & $\mathrm{AG}$ & 41164 & bois & $1650 \pm 30$ & $390-420 \mathrm{AD}$ & $3 \mathrm{AD}$ \\
\hline $\begin{array}{l}\text { Champetienne } \\
\text { coupe JG }\end{array}$ & s en MO & $\mathrm{AG}$ & Ly-34072 & charbon & $485 \pm 30$ & $1420-14$ & 1406-1451 AD \\
\hline tienne & CB. Alluvions limo & $\mathrm{AG}$ & Beta 341163 & $\begin{array}{l}\text { Sédiment } \\
\text { organique }\end{array}$ & $590 \pm 30$ & $1310-1400 \mathrm{AD}$ & $1299-1413 \mathrm{AD}$ \\
\hline $\begin{array}{l}\text { D.240 aval Cham- } \\
\text { petienne }\end{array}$ & C. Colluvions argilo-sableuses riches & $\mathrm{AG}$ & $\begin{array}{l}\text { 07S } 120 \\
\text { (via BRGM) }\end{array}$ & charbon & $1620 \pm 40$ & $389-534 \mathrm{AD}$ & $345-$ \\
\hline La Séauve & CB. Alluvions sablo-limoneuses & $\mathrm{AG}$ & Beta 392864 & $\begin{array}{l}\text { Matière } \\
\text { carbonée }\end{array}$ & $1210 \pm 30$ & $\mathrm{AD}$ & $695-8$ \\
\hline La Séauve & $\begin{array}{l}\text { CB. } \\
\text { chart }\end{array}$ & $\mathrm{AG}$ & Beta 392862 & $\begin{array}{l}\text { Matière } \\
\text { carbonée }\end{array}$ & $820 \pm 30$ & $1210-1255 \mathrm{AD}$ & $1165-1265 \mathrm{AD}$ \\
\hline Besseyro & blo-limoneuses & $\mathrm{AG}$ & 41157 & charbon & $1660 \pm 30$ & $\mathrm{AD}$ & $\mathrm{AD}$ \\
\hline Besseyrolle Basse & CB. Al & $\mathrm{AG}$ & Ly-11188 & charbon & $1710 \pm 30$ & $1690-1564 \mathrm{AD}$ & 251-397 AD \\
\hline Longetrée & $\begin{array}{l}\text { Ivions limono-sableuses à t } \\
\text { tion }\end{array}$ & $\mathrm{AG}$ & Poz-70707 & charbon & $1750 \pm 30$ & 245-332 AD & $222-385 \mathrm{AD}$ \\
\hline $\begin{array}{l}\text { Chartreuse de Bon- } \\
\text { nefoy }\end{array}$ & CB. Dépôt organique à nombreux débris de bois & AG & $\begin{array}{l}\text { 07S-128 } \\
\text { (via BRGM) }\end{array}$ & bois & $240 \pm 40$ & $1530-1940 \mathrm{AD}$ & $1520-1928 \mathrm{AD}$ \\
\hline $\begin{array}{l}\text { Chartreuse de Bon- } \\
\text { nefoy }\end{array}$ & CB. Dépôt argilo-sableus à blocs & AG & \begin{tabular}{|l}
$\begin{array}{l}\text { 07S-122 } \\
\text { (via BRGM) }\end{array}$ \\
\end{tabular} & bois & \pm 40 & 1416-1450 AD & $1330-1609 \mathrm{AD}$ \\
\hline $\begin{array}{l}\text { Chartreuse de Bon- } \\
\text { nefoy }\end{array}$ & CB. Dépôt argilo-sableus à blocs & $\mathrm{AG}$ & $\begin{array}{l}\text { 07S-125 } \\
\text { (via BRGM) }\end{array}$ & bois & $590 \pm 40$ & 1310-1406 AD & $1296-1416 \mathrm{AD}$ \\
\hline Arsac & CB. & $\mathrm{AG}$ & Ly-6447 & charbon & $2820 \pm 40$ & $\mathrm{BC}$ & $5 \mathrm{BC}$ \\
\hline Bouteyre (vallon) & CB. Alluvions & $\mathrm{AG}$ & Beta 367796 & bois & $400 \pm 30$ & 1450-1480 AD & $1436-1625 \mathrm{AD}$ \\
\hline Le Mazel & CB. Alluvio & $\mathrm{AG}$ & Ly-34074 & charbon & $560 \pm 30$ & 1322-1416 AD & $1307-1429 \mathrm{AD}$ \\
\hline $\begin{array}{l}\text { Champetienne Log } \\
\text { T1haut }\end{array}$ & CT. Colluvions granitiques & TOR & Beta 386222 & charbon & $1940 \pm 30$ & $30-80 \mathrm{AD}$ & $20 \mathrm{BC}-130 \mathrm{AD}$ \\
\hline $\begin{array}{l}\text { Champetienne T1 } \\
\text { Log M6 }\end{array}$ & CT. Alluvions argilo-sableuses gleyiques & TOR & Beta 386230 & charbon & $1850 \pm 30$ & $125-220 \mathrm{AD}$ & $85-235 \mathrm{AD}$ \\
\hline $\begin{array}{l}\text { Champetienne T1 } \\
\text { Log } 3-4 \mathrm{~m}\end{array}$ & CT. Alluvions sableuses & TOR & Beta 386224 & charbon & $570 \pm 30$ & $1320-1410$ AD & $1304-1423 \mathrm{AD}$ \\
\hline $\begin{array}{l}\text { Champetienne T1 } \\
\text { Log3-4m }\end{array}$ & CT. & TOR & 27 & ch & 30 & $\mathrm{AD}$ & $9 \mathrm{AD}$ \\
\hline $\begin{array}{l}\text { Champetienne T1 } \\
\text { Log3-4m }\end{array}$ & CT. Alluvions sablo-limoneuses riches en MO & TOR & Beta 386228 & charbon & $90 \pm 30$ & $\begin{array}{l}1695- \\
\text { post } 1950 \mathrm{AD}\end{array}$ & $1685-1928 \mathrm{AD}$ \\
\hline Gage La Valette & CB. Alluvions sablo-limoneuses oxydées & TOR & Beta 392853 & charbon & $320 \pm 30$ & 1500-1640 AD & 1483-1646 AD \\
\hline
\end{tabular}


la base de données n'est le plus souvent pas représentative d'un point de vue statistique et des études ont démontré qu'une base de données radiocarbone doit comporter au minimum 20 individus pour présenter un diagramme de probabilités fiable (Williams, 2012) ; dans le cas contraire, les creux ou hiatus chronologiques sont sujets à caution. D'autres auteurs affirment que 50 à 100 dates sont le minimum nécessaire à la constitution d'une base de données solide (Michczynska et al., 2007). Les limites énumérées au niveau du site peuvent être contournées et corrigées à l'échelle régionale par un échantillonnage aléatoire lorsqu'un large assemblage de sites datés par le radiocarbone est disponible (Berger, 2015). La moyenne de la déviation standard de la date radiocarbone brute apparaît comme l'élément déterminant la qualité de l'échantillon analysé. Les écarts-types trop larges doivent donc être éliminés de l'analyse. Les dates présentant un écart-type supérieur à 150-200 ans BP sont considérées comme non représentatives, surtout lorsque l'on souhaite, comme c'est le cas ici, mettre en évidence des variations environnementales de l'ordre de quelques décennies ou du siècle. Les âges obtenus au cours de la dernière décennie, issus de l'amélioration des méthodes de datation radiocarbone (par accélérateur, notamment) et des méthodes de calibration, permettent de réduire le nombre d'échantillons des séries radio-chronologiques analysables. La plupart des dates présentent aujourd'hui un écart-type standard de 30 à 40 années BP.

Les effets de calibration sont également importants. Ils sont associés aux variations atmosphériques du taux de radiocarbone et à l'activité du soleil; ils sont donc intrinsèques à la méthode. Les effets des plateaux radiocarbone peuvent ainsi élargir un événement radiocarbone en une longue période chronologique, ou le réduire dans les cas où un raidissement de la courbe de calibration est observé, plusieurs dates étant alors assimilées à un simple pic temporellement très court (Berger, 2015). Si aucune méthode statistique ne semble capable de corriger ce biais de la méthode, l'ajout de diagrammes en bâtons indiquant le nombre de dates responsables de chaque pic permet cependant de relativiser leur importance les uns par rapport aux autres (Williams, 2012).

Enfin, d'autres limites à cette généralisation de l'utilisation de l'analyse CPDF sont moins en rapport avec la structure des bases de données ou avec la production du radiocarbone qu'avec le contexte et le type de matériaux utilisés (Berger et al., 2008 ; Berger, 2015).

Une analyse statistique exploitant le panel des résultats géochronologiques et litho-stratigraphiques obtenus dans l'ensemble du bassin supérieur de la Loire a donc été conduite afin de caractériser à l'échelle micro-régionale les principales phases de l'évolution hydro-sédimentaire.

Tous les résultats radiocarbone ont été calibrés en âges BP avec le logiciel Calib 7.01 pour standardiser les résultats. Nous avons choisi de travailler sur la base de la fourchette de calibration à $1 \mathrm{~s}(68,2 \%)$, notamment pour affiner le phasage des fluctuations dans les périodes les plus documentées du dernier millénaire. Une macro d'Excel a été utilisée pour construire la courbe de probabilité cumulée sur laquelle l'axe des abscisses représente l'âge radiocarbone calibré et l'axe des ordonnées la probabilité de l'événement par an (Manen \& Sabatier, 2003). Le logiciel utilisé permet de cumuler des dates radiocarbone calibrées avec des événements dont la chronologie est estimée entre les centres des écarts-types de deux dates radiocarbone, ou même à l'aide d'une chronologie culturelle. Il pondère chaque date en fonction de la durée de sa fourchette de calibration et/ou de son attribution chrono-culturelle (la surface du rectangle représentant la date, restant identique). Cela accroît ainsi le poids des datations les plus précises et dilue celui des moins précises (Evin et al., 1995). Les principaux pics de la courbe discutés dans cet article sont supérieurs à 3 . Les pics secondaires compris entre 1 et 2 et les pics mineurs inférieurs à 1 ne sont pas discutés car créés par moins de 3 dates. Chaque unité représentée sur l'axe des ordonnées (fig. 3, de 0 à 12) correspond donc théoriquement à un cumul de 4 à 8 dates, marqué par le regroupement des bâtonnets matérialisant le centre de chaque date calibrée.

Les quelques plateaux radiocarbone qui jalonnent le dernier millénaire, notamment lors des variations de l'activité du soleil synchrones du Petit Âge Glaciaire (PAG), n'ont semble-t-il pas trop perturbé le résultat de l'analyse CPDF car les écarts-type du corpus de résultats SMA utilisés demeurent très resserrés. Pour les dates radiocarbone brutes inférieures à $250 \mathrm{BP}$, nous avons fait le choix de ne pas utiliser le dernier pic de probabilité de la calibration, qui correspond la plupart du temps aux premières décennies du $\mathrm{Xx}^{\mathrm{e}}$ siècle jusqu'à 1950 , car les contextes chrono-stratigraphiques étudiés permettent de le rejeter (il s'agit de la datation de dépôts tous antérieurs à leur incision par le système fluvial, qui s'est amorcée à la fin du XIX ${ }^{e}$ siècle).

\section{3 - RÉSULTATS}

Nous présentons ici les résultats de l'application de la méthode CDPF aux séquences litho-stratigraphiques caractérisées pour les trois derniers millénaires (fig. 3). Seule cette période, et surtout les deux derniers millénaires, bénéficie en effet d'un cortège suffisant de datations réparties entre différents sites pour permettre une analyse statistiquement robuste d'où ressort un phasage de l'évolution à valeur micro-régionale. Les douze phases déduites de cette analyse sont caractérisées sous l'angle des dynamiques hydro-pédo-sédimentaires.

\section{1 - PHASE $1: 1^{\text {er }}$ MILLÉNAIRE AV. J.-C.}

Cette période est marquée majoritairement par des phases d'hydrologie active, notamment au cours du $\mathrm{IV}^{\mathrm{e}}$ siècle av. J.-C. (Tène ancienne ?). Elle est cependant encore trop peu documentée pour en préciser les caractères. 


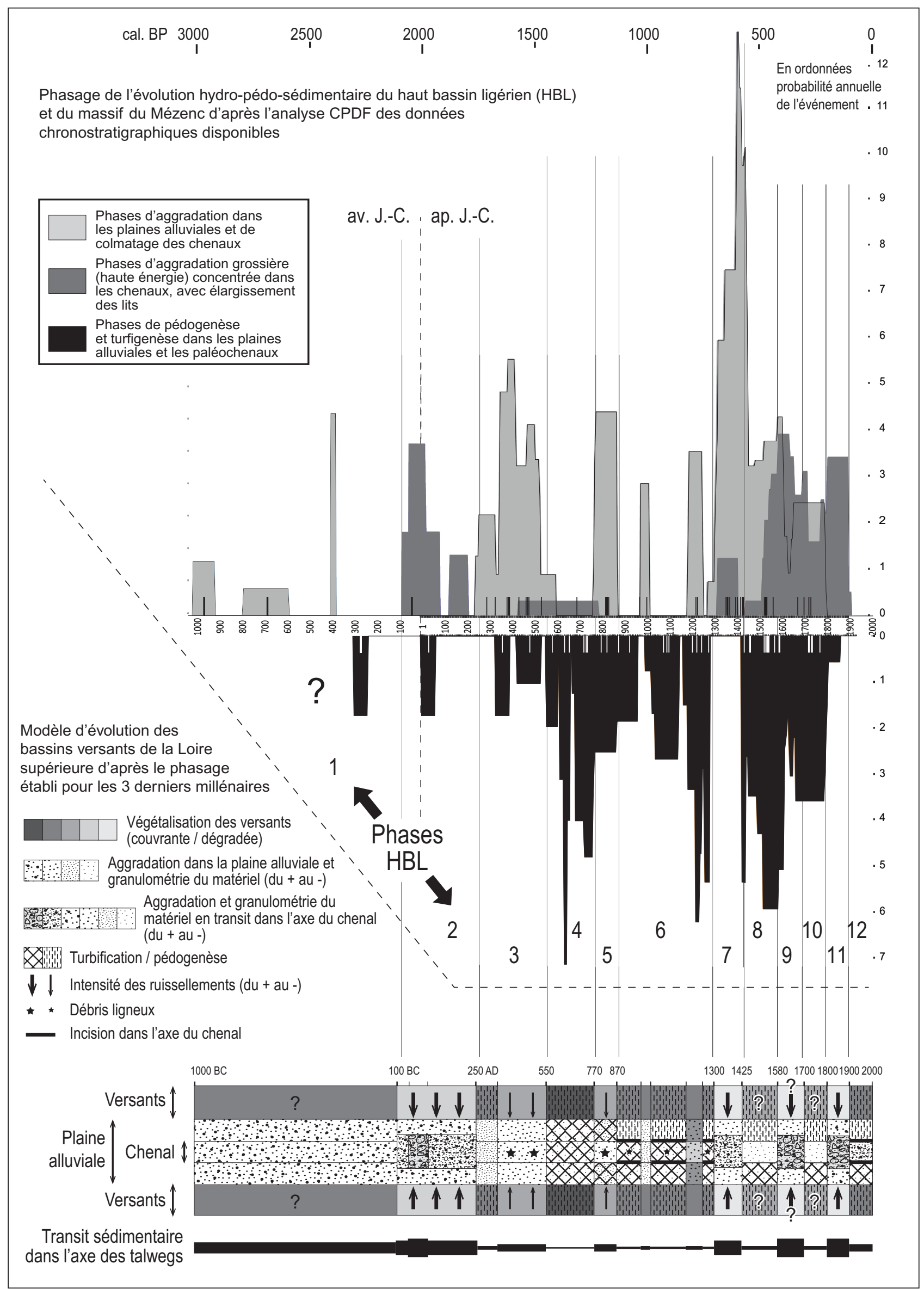

Fig. 3 : Phasage de l'évolution hydro-pédo-sédimentaire du haut bassin ligérien (HBL) et du massif du Mézenc d'après l'analyse CPDF des données chrono-litho-stratigraphiques disponibles et modèle d'évolution établi pour les bassins versants à l'échelle des trois derniers millénaires. Fig. 3: Hydro-pedo-sedimentary evolution stages in the upstream Loire basin (HBL) and the Mézenc massif, based on CPDF analysis of available chrono-litho-stratigraphic data and evolution model for headwaters basins for the last three millenia. 


\section{2 - PHASE 2 : DE 100 AV. J.-C. À 250 AP. J.-C.}

Des écoulements concentrés de haute énergie, culminant autour de 0 av./ap. J.-C., affectent les axes fluviaux et les versants. La connectivité amont-aval semble maximale, alors que les conditions de drainage se dégradent en fond de vallon (indices d'hydromorphie).

\section{3 - PHASE 3 : DE 250 À 550 AP. J.-C.}

Suite à la crise érosive de la phase précédente, les dynamiques hydro-sédimentaires s'atténuent. Elles sont à l'origine de dépôts réguliers de crues d'énergie modérée. Des phases d'aggradation sablo-limoneuse rapide (sols cumuliques), chargées en débris ligneux et en macro-restes végétaux dans l'axe des chenaux alluviaux, témoignent d'un probable décapage des litières pédologiques en amont. La première partie de cette phase (fin $\mathrm{III}^{\mathrm{e}}-\mathrm{IV} \mathrm{V}^{\mathrm{e}}$ siècle) est moins détritique et de plus faible énergie, associée à des pédogenèses dans un contexte assez hydromorphe. Après cette période d'accalmie relative et à partir de la deuxième moitié $d u$ Iv ${ }^{\mathrm{e}}$ siècle, le détritisme et l'énergie des écoulements s'accroissent de nouveau.

\section{4 - PHASE 4 : DE 550 À 770 AP. J.-C.}

Il s'agit d'une phase de stabilisation maximale sur les versants et dans les plaines alluviales avec colmatage des lits fluviaux et des dépressions par des formations organogènes authigènes (pédogenèse, turfigenèse). Quelques épisodes plus minérogènes (sables et limons) illustrent de courtes périodes d'instabilité fluviale. Le transit sédimentaire depuis l'amont est nettement réduit par rapport aux premiers siècles de ce millénaire (Haut-Empire romain) et les conditions de drainage se dégradent (surtout dans la deuxième partie de cette période).

\section{5 - PHASE 5 : DE 770 À 870 AP. J.-C.}

Cette phase séculaire de dérèglement hydro-sédimentaire est identifiée simultanément dans plusieurs bassins versants. L'augmentation forte du détritisme s'accompagne malgré tout d'une poursuite des processus de turfigenèse. Les signatures torrentielles sont absentes et les aggradations sont nourries par des flux de matériaux fins chargés en matière organique et en débris de litières, à l'origine de sols cumuliques dans un contexte très hydromorphe.

\section{6 - PHASE 6 : DE 870 À 1300 AP. J.-C.}

Cette période se caractérise par une très forte réduction des flux hydro-sédimentaires à l'échelle du haut bassin ligérien. Les processus de pédogenèse y prédominent (paléosols alluviaux ou formations tourbeuses). Les lits enregistrent une sédimentation de faible énergie associée à des dépôts fins souvent chargés en matière organique. Il est difficile de parler d'encaissement synchrone des lits, car les données stratigraphiques sont rares, mais l'hypothèse est plausible, notamment à partir des données recueillies dans le vallon de Champetienne (Defive et al., 2015) (fig. 1 et 2). Dans cette tendance à la stabilisation des lits fluviaux, deux courts épisodes d'aggradation sédimentaire surviennent, vers 975-1020 ap. J.-C. environ (épisode clairement associé alors à une baisse des indices pédogénétiques) et vers 1180-1250 ap. J.-C.

\section{7 - PHASE 7 : DE 1300 À 1425 AP. J.-C.}

Cette période est marquée par un retour des processus d'aggradation dans les plaines alluviales avec, ponctuellement, des épisodes de plus haute énergie (dépôts plus torrentiels). Ce basculement, opéré durant le $\mathrm{XIV}^{\mathrm{e}}$ siècle à l'échelle du haut bassin ligérien, est également marqué par une forte augmentation des flux de matières en suspension (MES), suggérant une importante phase d'érosion des sols.

\section{8 - PHASE 8 : DE 1425 À 1580 AP. J.-C.}

Un nouveau basculement de la tendance morphogénique, brutal et homogène, survient à la fin du premier quart $\mathrm{du} \mathrm{Xv}^{\mathrm{e}}$ siècle. La stabilisation des cours d'eau est suggérée par une diminution rapide des épisodes de crue et d'aggradation dans l'axe des chenaux et par l'accumulation de tourbe dans les points bas, alors que les processus pédogéniques s'impriment sur les alluvions antérieures dans les autres secteurs des petites plaines alluviales des têtes de bassin. Cette période de stabilité se termine dans les dernières décennies du XVI ${ }^{e}$ siècle par un retour progressif à des épisodes hydro-sédimentaires plus énergiques.

\section{9 - PHASE 9 : DE 1580 À 1700 AP. J.-C.}

Cette phase est marquée par un retour des indices d'écoulements de très haute énergie dans les lits fluviaux (formations torrentielles). Les signatures pédogéniques marquent alors un creux prononcé sur la courbe CPDF, révélant la forte instabilité des lits fluviaux et la fréquence des apports sédimentaires grossiers ou sableux laminés.

\subsection{0 - PHASE 10 : DE 1700 À 1800 AP. J.-C.}

Il s'agit d'un épisode séculaire de forte remontée des indices de pédo- et turfigenèse, avec diminution corrélative des épisodes de forte activité hydro-sédimentaire (moindre torrentialité). Les faciès plus fins (MES) sont alors assez bien représentés et témoignent de dépôts de crue plus fins et irréguliers associés à la turfigenèse dans les points bas et les parties distales des plaines alluviales. Le $\mathrm{XVIII}^{\mathrm{e}}$ siècle semble témoigner ainsi à l'échelle de la région d'étude d'une diminution de l'activité hydrologique et de l'érosion des sols.

\subsection{1 - PHASE 11 : DE 1800 À 1900 AP. J.-C.}

Le retour et la prédominance des faciès grossiers, associés à des dynamiques torrentielles, s'opère à la transition $\mathrm{XVIII}^{\mathrm{e}}-\mathrm{XIX}^{\mathrm{e}}$ siècle et durant tout le $\mathrm{XIX}^{\mathrm{e}}$ siècle. Les indicateurs de turfi- et pédogenèse disparaissent 
alors complètement, comme les dépôts d'aggradation fins. Ces formations gravelo-caillouteuses grossières coiffent une majorité des formations holocènes récentes, préalablement à l'incision subactuelle/actuelle. Elles témoignent d'une gradation positive d'énergie au cours de la première moitié de la période contemporaine, lors d'un maximum démographique et d'altération du couvert végétal en moyenne montagne. Les connectivités longitudinale et latérale semblent alors maximales dans les têtes de bassins versants ligériens (dépassant celles du Haut-Empire romain), favorisant les phénomènes de torrentialité et les impacts sur les aménagements humains de fond de vallée.

\subsection{2 - PHASE 12 : DE 1900 AP. J.-C. À L'ACTUEL}

Cette dernière phase correspond à une absence de dépôts datés sur la courbe de CPDF, corroborée par la tendance à l'incision dans l'ensemble des sous-bassins versants étudiés. La période précise d'inversion de la dynamique fluviale est encore sujette à discussion, mais l'âge de certains arbres des ripisylves perchées entre 1 et $3 \mathrm{~m}$ au-dessus des lits fluviaux actuels peut être estimé à plus d'un siècle.

\section{4 - DISCUSSION}

Le premier millénaire av. J.-C. est encore trop peu documenté dans les archives fluviales du bassin supérieur de la Loire pour être sérieusement commenté. L'activité hydrologique qui s'y manifeste coïncide avec une période de péjoration climatique (Dansgaard et al., 1969 ; Schönwiese, 1995 ; Holzhauser et al., 2005), mais aussi avec une période de défrichements dont l'impact est enregistré dans les tourbières du secteur étudié (Couteaux, 1984).

Comme dans la haute et moyenne vallée du Rhône (Bravard et al., 1992 ; Allée, 2003 ; Berger, 2015), l'activité hydro-sédimentaire des têtes de bassin de la Loire supérieure apparaît extrême entre 100 av. J.-C. et 250 ap. J.-C. Elle est alors associée dans ces micro-régions à une dynamique torrentielle généralisée, que confirment les sources historiques (Defive et al., 2015, annexe 15 p. 54). Notre lecture n'est pas encore suffisamment fine dans le haut bassin ligérien pour comprendre comment s'établit le lien entre cette histoire et le contexte climatique connu par ailleurs (250 av. J.-C. - 400 ap. J.-C. : RWP - Roman Warm Period ou Optimum Climatique Romain, marquant essentiellement le Haut-Empire). Dans les Alpes du Nord (Arnaud et al., 2012), comme dans le bassin rhodanien moyen (Berger \& Bravard, 2012 ; Notebaert \& Berger, 2014), l'hypothèse d'un forçage anthropogénique majeur et sans équivalent holocène, des plaines aux massifs montagnards, a été discutée sur la base d'études systémiques, géochimiques à haute résolution et semi-quantitatives (budgets sédimentaires). Dans le haut bassin ligérien, les données historiques et archéologiques disponibles (Dendievel, 2012) ainsi que la palynologie
(Couteaux, 1984 ; de Beaulieu et al., 1984) témoignent d'une intensification de la présence humaine et avec elle d'un impact croissant sur l'environnement végétal (importants défrichements liés à l'intensification du pastoralisme, extension de la culture des céréales jusque sur les plus hautes terres) et sur les dynamiques érosives (Rhoujjati, 1995 ; Defive et al., 2015). Certains sites témoignent d'une présence humaine en altitude plus ancienne encore, perceptible dans les diagrammes dès le $\mathrm{IV}^{\mathrm{e}}$ siècle av. J.-C. avec des indices de défrichements (par exemple à La Narce sur la commune du Béage, Dendievel et al., 2014, 2015, 2016 ; fig. 1).

La période de débits renforcés identifiée à la charnière Antiquité - Moyen Âge entre le Iv ${ }^{\mathrm{e}}$ et le milieu $\mathrm{du} \mathrm{VI}^{\mathrm{e}}$ siècle ap. J.-C. coïncide approximativement avec celle qui a été identifiée dans le bassin moyen du Rhône (Notebaert et al., 2014 ; Notebaert \& Berger, 2014 ; Salvador \& Berger, 2014 ; Berger, 2015), dans le Limousin (Allée, 2003) et dans la région lyonnaise (Bravard et al., 1997). Elle apparaît cependant légèrement décalée, car elle s'amorce un peu plus tôt dès le $\mathrm{IV}^{\mathrm{e}}$ siècle et s'achève un peu plus tôt dans $\mathrm{le}_{\mathrm{VI}}^{\mathrm{e}}$ siècle (début $\mathrm{du} \mathrm{VII}^{\mathrm{e}}$ siècle dans l'aire rhodanienne). Les données issues de l'étude de séquences tourbeuses du plateau du Béage (dépression de La Narce et tourbière de pente de Pialeloup), à l'extrémité sud du massif du Mézenc dans le haut bassin de la Loire (fig. 1) corroborent cette phase d'instabilité et suggèrent le rôle que l'homme a pu jouer dans l'accroissement de l'érosion. Sur ces sites, les indices paléoécologiques (macrorestes et palynologie) signalent une érosion accrue sur les versants, la présence d'épisodes de feu soutenus (défrichements) ainsi qu'une mise en culture de la zone depuis l'Antiquité jusqu'au $\mathrm{VI}^{\mathrm{e}}$ siècle ap. J.-C. (Dendievel et al., 2014, 2016). Cette période d'augmentation de l'activité hydro-sédimentaire a aussi été mise en évidence dans de petits bassins versants plus en aval du bassin amont de la Loire (Limagne d'Allier : Ballut \& Trément, 2006).

Le haut Moyen Âge est marqué par l'instabilité du fonctionnement des hydrosystèmes élémentaires du haut bassin ligérien, alternant périodes d'exacerbation de l'érosion et périodes d'accalmie. La phase 4 (550770 ap. J.-C.) de l'analyse CPDF correspond ainsi à une période de stabilisation prononcée des milieux. Cette tendance semble enregistrée également, mais plus précocement (juste avant 428-660 ap. J.-C. ; $1470 \pm 60$ BP), sur le plateau du Devès immédiatement à l'ouest de la zone étudiée (de Beaulieu et al., 1984) où est enregistrée une phase de recul assez net de la pression anthropique et de fermeture du milieu (très fort développement des taxons pionniers héliophiles comme le Pin, déterminant un faciès de reconquête de terres abandonnées ou moins intensément exploitées). La question se pose du rôle des facteurs historiques dans le contrôle de cette phase d'accalmie, au cours d'une période où sont identifiés dans les moyennes montagnes auvergnates quelques foyers d'occupation humaine dans une phase de baisse démographique régionale (Trément et al., 2014) qui ne semble pas avérée dans le cas du haut bassin ligérien (Dendievel, 2012). 
Au contraire de la phase 4, la phase 5 (770-870 ap. J.-C.) est marquée par un dérèglement à l'origine de pulsations détritiques tandis que se poursuit la constitution d'accumulations organiques. La relation entre ce regain séculaire de la torrentialité en contexte de moyenne montagne (fig. 3 : phase 5) et une courte phase bien marquée de progradation des glaciers alpins (Ivy-Ochs et al., 2009 ; Le Roy et al., 2015) reste en discussion, une période de rafraîchissement ayant pu abaisser la limite supérieure des forêts. Un pic d'activité hydro-sédimentaire est également observé autour du lac d'Anterne dans les Alpes du Nord à plus de $2000 \mathrm{~m}$ d'altitude (Giguet-Covex et al., 2011). Ces tendances s'expriment alors que les sources historiques et archéologiques montrent la multiplication des sites d'occupation à l'échelle du haut bassin ligérien, le haut Moyen Âge ne connaissant pas ici de chute démographique (Dendievel, 2012).

La phase 6 de l'analyse CPDF correspond au POM (Petit Optimum Médiéval), les phases 7 à 11 au PAG (Petit Âge Glaciaire), avec deux périodes de diminution de l'activité hydro-sédimentaire (phases 8 et 10) (fig. 3). Le POM se caractérise sur la plupart des courbes CPDF publiées à l'échelle de l'Europe moyenne par un creux marqué de l'activité hydro-sédimentaire centré sur le $\mathrm{x}^{\mathrm{e}}$ siècle, de durée plus ou moins longue. Il est court en Angleterre (Büntgen et al., 2011 ; Macklin et al., 2010) et dans le bassin de la Loire (2 à 3 siècles ; Castanet, 2008). Il est plus long et plus marqué dans les hauts et moyens bassins du Rhône et dans le bassin de la Vistule (du VIII ${ }^{\mathrm{e}}$ au XII ${ }^{\mathrm{e}}$ siècle) et caractérisé par un fort développement pédologique dans les plaines alluviales rhodaniennes (Berger, 2006, 2015). La tendance observée dans le haut bassin ligérien se rapproche fortement de celle du bassin rhodanien. Malgré la rareté encore prononcée des données archéologiques disponibles dans ce secteur, la question se pose du rôle de l'anthropisation dans la stabilisation des milieux durant cette période. Les recherches engagées sur le bassin versant de Champetienne (Fournier, 2014) et sur le vallon de Mazan (Lugan, 2014) démontrent une densité certaine des occupations humaines et des équipements agropastoraux et hydrauliques des versants et des fonds de vallée. En croisant les sources planimétriques anciennes (carte de Cassini, cadastre dit napoléonien), les indices toponymiques, l'analyse du parcellaire, les formes du paysage et quelques prospections de terrain, il est possible de mettre en évidence d'anciens systèmes mixtes alliant drainage, irrigation et exploitation de la force hydraulique, ainsi que des aménagements liés au pacage des troupeaux (enclos, délimitations parcellaires) et à la culture (terrasses fossiles). À Mazan (fig. 1), les structures mises en évidence peuvent être corrélées à l'occupation monastique qui intervient dès le XII ${ }^{\mathrm{e}}$ siècle et perdure jusqu'à la Révolution française (Lugan, 2014 ; Bouvard, 2016). Le même constat a été établi à la Chartreuse de Bonnefoy près du Béage (fig. 1), où de nombreuses structures bâties, notamment hydrauliques (moulins, viviers, barrages, digues) subsistent encore à l'état de ruines ou de paléoreliefs. Le contrôle de l'eau dans les bassins versants apparaît ainsi essentiel, son impact sur le transit des flux-hydrosédimentaires restant à préciser.

La courbe CPDF construite pour le haut bassin ligérien apporte une nouvelle contribution démontrant que le PAG représente un épisode climatique polyphasé, dont les manifestations hydro-géomorphologiques ont été fortement influencées par les forçages climatiques (paramètres pluviométriques et thermiques) et anthropiques (types de mises en valeur, impacts sur la végétation et les sols favorisant les connectivités à l'échelle des bassins versants, cycles d'emprise/déprise). Cette période est bien documentée dans la littérature par les recherches hydro-géomorphologiques car son occurrence comparativement récente a favorisé la surreprésentation de ses archives par rapport aux analogues plus anciens de dégradation hydro-climatique, notamment dans les fonds de vallées fluviales qui ont subi d'importantes modifications de leur lit au cours de derniers siècles très torrentiels (Bravard, 2010). Les archives du haut bassin ligérien confirment le très bon état de l'enregistrement hydro-géomorphologique pour les derniers siècles.

Il est classiquement admis que le PAG correspond à la période pendant laquelle les glaciers avancent de façon globale, au début du XIV siècle (Grove, 2001). Mais si on le fait débuter au moment où l'instabilité climatique commence, il débute à la fin du XIII ${ }^{\mathrm{e}}$ siècle (même vers 1280 pour Grove, 2001 ou Holzhauser, 1997 ; 1315 pour Le Roy Ladurie, 2004 et 1303 pour Le Roy Ladurie et al., 2011). Cette première avancée glaciaire importante culmine dans les Alpes vers 1350 ap. J.-C. (Zumbühl \& Holzhauser, 1988 ; Holzhauser et al., 2005). En termes de bilan hydrique, le PAG marque dans le bassin supérieur de la Loire un accroissement sensible des apports hydriques, qui correspond aussi à une transgression lacustre marquée dans les Alpes du Nord et le Jura (Chapron et al., 2002 ; Magny, 2004 ; Debret et al., 2010 ; Magny et al., 2010).

La première manifestation du PAG (PAG 1) est ainsi bien représentée dans les archives du haut bassin ligérien (fig. 3 : phase 7 de l'analyse CPDF), l'épisode de réchauffement et d'assèchement intra-PAG à travers la phase 8 et le second PAG (ou pléni-PAG) par les phases 9 à 11 . La stabilité hydro-sédimentaire identifiée entre le milieu $\mathrm{du} \mathrm{Xv}$ siècle et la fin du Xvie siècle dans le bassin ligérien (phase 7) correspond à une phase séculaire d'incision des lits fluviaux dans le Rhône moyen (Berger, 2015), mais aussi à une baisse des apports de crue du Rhône dans le lac du Bourget (Arnaud et al., 2008), de l'activité hydro-sédimentaire dans les principales rivières du sud de l'Angleterre (Macklin et al., 2010), et au développement d'une activité pédogénétique centrée sur 1500 ap. J.-C. dans les haut et moyen bassins du Rhône (Berger, 2015) ainsi qu'à l'échelle de l'Allemagne (Hoffmann et al., 2008a,b).

La partition du PAG a été récemment discutée (Berger, 2015), dans la lignée des travaux menés sur les archives lacustres nord-alpines (le Bourget) et jurassiennes (lac de Saint-Point) (Magny et al., 2010). Au lac de Saint-Point, les bas niveaux lacustres à tourbe datés coïncident avec des phases de forte activité solaire (au XII ${ }^{\mathrm{e}}$ siècle, vers 1630,1760 et depuis la fin du XIX ${ }^{e}$ siècle), tandis que les 
phases de haut niveau lacustre en anti-corrélation sont synchrones des phases de faible activité solaire (Magny et al., 2010). La mise en parallèle des données acquises sur trois marqueurs différents dans des environnements variés (fluctuations du glacier d'Aletsch, fluctuations lacustres à Saint-Point, flux détritiques du paléo-Rhône au Bourget) démontre à l'échelle des Alpes occidentales et du Jura un forçage climatique identique au cours du PAG, sous contrôle apparemment solaire (Magny et al., 2010).

Une divergence ressort du cadre climatique présenté par Magny et al. (2010), qui n'a pas été discutée par les auteurs : un retrait glaciaire important intra-PAG, entre 1430 et 1580 , n'apparaît pas en phase avec les indicateurs lacustres et détritiques discutés. Il correspond, par contre, aux variations enregistrées dans le domaine fluvial du Rhône moyen (Berger, 2015), de la Durance (Miramont \& Guilbert, 1997), dans les hydrosystèmes de Russie occidentale (Berger, 2015) et dans les têtes de bassin ligériennes (ce travail). Le Roy Ladurie (2004) évoque un " beau XVI ${ }^{e}$ siècle » à l'échelle de l'Europe occidentale entre 1500 et 1560. Büntgen et al. (2011) révèlent, sur la base d'une imposante synthèse dendro-climatologique des Alpes centrales, une période plus sèche et plus chaude entre 1400 et 1575 . Nous retrouvons enfin le même schéma tripartite dans la dynamique du glacier d'Aletsch, marquée par une régression glaciaire affirmée entre 1400 et 1600 , entre deux périodes de poussée glaciaire (Holzhauser et al., 2005). La tripartition du PAG apparaît ainsi clairement à l'échelle de l'Europe occidentale, en considérant la dynamique des lacs, des glaciers d'altitude, celles du bassin moyen du Rhône (Berger, 2015), du Rhin (Hoffmann et al., 2008a,b), de la Durance (Miramont \& Guilbert, 1997) et du haut bassin ligérien (Defive et al., 2015).

Outre ces corrélations inter-régionales à l'échelle des piémonts alpins et de leurs avant-pays, la présente étude met en évidence un second court épisode de diminution de l'activité hydro-sédimentaire au cours de la période considérée comme la plus active du PAG dans les archives sédimentaires et glaciaires continentales. Cet épisode, marqué par une forte pression des populations montagnardes, survient au cours de la phase 10, c'està-dire au $\mathrm{XVIII}^{\mathrm{e}}$ siècle. Ce siècle enregistre par ailleurs plusieurs courtes phases de retrait glaciaire dans les Alpes occidentales entre 1680 et 1810 au cours d'une période d'avancée glaciaire prolongée (Nussbaumer et al., 2011). La dynamique d'érosion semble également se réduire plus ou moins fortement dans les bassins versants de plusieurs lacs alpins (Blanc, Anterne, Allos) entre 1660/1700 et 1780 (Wilhelm et al., 2012; Arnaud et al., 2016). L'ensemble de ces convergences pourrait bien signer une phase de moindre agressivité des conditions climatiques sur les environnements de moyenne et haute montagne de l'Europe occidentale.

Si des liens évidents apparaissent ainsi à l'échelle du cycle POM-PAG entre fluctuations climatiques et variation des flux hydro-sédimentaires sur les versants et dans l'axe des talwegs du haut bassin ligérien, l'impact envi- ronnemental de l'anthropisation reste à mesurer localement. Il est probable que le schéma soit ici conforme à celui qui a par ailleurs été mis en évidence par Allée (2003) en Limousin et au Mont Lozère : le climat détermine un potentiel érosif, tandis que la répartition spatiale des activités humaines détermine les lieux où ce potentiel s'exprimera effectivement, ainsi que le degré d'intensité de la réponse érosive. Pour atteindre ce niveau de résolution dans l'analyse, les recherches demandent à être poursuivies dans le bassin supérieur de la Loire où seules les grandes tendances de l'histoire de l'occupation humaine sont connues et où la spatialisation des données demeure très incomplète. Retenons tout de même que le POM coïncide avec l'implantation sur ces hautes terres, surtout à partir du XII ${ }^{\mathrm{e}}$ siècle, de grands domaines ecclésiastiques et avec eux d'estives de grands troupeaux ovins transhumant entre la vallée du Rhône et les hauts plateaux du Mézenc (voir, entre autres, Régné, 1923 ; Cornu, 1991 ; Brechon, 1998, 2000 ; Blanc, 2000 ; Brechon, 2001 ; Bouvard, 2009, 2011, 2013, 2016). La constitution de noyaux d'habitat permanent (villages, hameaux) dominants en-dessous de $1200 \mathrm{~m}$ d'altitude débute sans doute dès avant le Moyen Âge, et se trouve pratiquement achevée dès le $\mathrm{XVI}^{\mathrm{e}}$ siècle. Elle est accompagnée à partir du XIV siècle d'un essaimage en fermes isolées autour des hameaux et sur les plus hautes terres, qui va s'intensifier au cours des XVII ${ }^{e}$ et XVIII ${ }^{e}$ siècle et jusqu'à l'apogée du XIX siècle (Fel, 1962 ; Molinier, 1985 ; Léogier, 1995, 2005 ; Dendievel, 2012). À l'ouest du massif du Mézenc, le diagramme pollinique de Landos (de Beaulieu et al., 1984) témoigne bien de cette conquête progressive des hautes terres avec, à partir du milieu du $\mathrm{Xv}^{\mathrm{e}}$ siècle, un signal fort et omniprésent des indicateurs de mise en culture et de pastoralisme. Dans le massif du Mézenc, l'impact environnemental de cette conquête des hautes terres doit être regardé avec prudence dans la mesure où ce massif a connu une spécialisation précoce (amorcée dès la fin du bas Moyen Âge) dans l'élevage bovin pour la production de viande et de bœufs de trait (Brechon, 1998, 2000, 2001). Sur les plus hautes terres au-dessus de $1200 \mathrm{~m}$, cette orientation concurrence l'élevage ovin transhumant dès le $\mathrm{XVII}^{\mathrm{e}}$ siècle puis le supplante par la suite. Ainsi, malgré un recul prononcé des surfaces forestières du fait des défrichements poursuivis au fil des siècles accompagnant le PAG et la présence de parcelles cultivées dans certains bassins versants, (Reumaux, 2002, 2003 ; Simandoux, 2007, 2008 ; Archives départementales de l'Ardèche, 2006), il n'est pas certain que la réponse érosive globale ait été ici aussi forte qu'ailleurs, notamment en raison de l'extension des herbages associés à cet élevage bovin pour la production de foin. D'après ce que nous connaissons de l'histoire du haut bassin de la Loire et du massif du Mézenc, c'est en fait au bas Moyen Âge que les labours semblent s'être le plus étendus en altitude, accroissant l'exposition des terres à l'érosion dans les premiers temps du PAG, comme le montre l'explosion des flux de MES responsables de rapides aggradations de matériaux fins dans les petites plaines alluviales de tête de bassin. 


\section{5 - CONCLUSION}

Les résultats obtenus dans le haut bassin de la Loire et qui documentent l'histoire hydro-sédimentaire des trois derniers millénaires s'appuient sur un nombre de datations suffisamment important (une fois éliminées les dates stratigraphiquement incohérentes liées à des remaniements de charbons) pour pouvoir être considérés comme fiables. Ils offrent cependant une lecture encore très inégale suivant les périodes, seuls les trois, et même essentiellement les deux, derniers millénaires donnant matière à reconstituer une séquence chronologique précise appuyée sur les données chrono-litho-stratigraphiques.

Des évidences stratigraphiques permettent de pointer la crise érosive qui survient au début du second âge du Fer, mais les datations sont encore trop peu nombreuses, de même que les sites recelant des dépôts de cet âge, pour permettre d'en préciser les termes. Les deux principales crises érosives postérieures se produisent sous l'effet d'un double forçage climatique et anthropique : d'une part au moment de l'Antiquité gallo-romaine avec un maximum il y a environ 2000 ans, puis entre le $\mathrm{XIV}^{\mathrm{e}}$ et la fin du XIX ${ }^{e}$ siècle. La connectivité amont-aval entre versants et talwegs est alors maximale au sein des bassins versants. Après la période pluriséculaire de stabilité du POM, cette dernière crise érosive apparaît dans le bassin supérieur de la Loire conforme à la tripartition du PAG connue par ailleurs (fort hydro-dynamisme aux XIV et début du $\mathrm{Xv}^{\mathrm{e}}$ siècles, puis accalmie jusqu'à la fin $\mathrm{du} \mathrm{XvI}^{\mathrm{e}}$ siècle, puis retour à un très fort hydrodynamisme jusqu'à la fin du $\mathrm{XIX}^{\mathrm{e}}$ siècle). Une période supplémentaire de biostasie relative, inédite, s'y manifeste au XVIII ${ }^{\mathrm{e}}$ siècle. Si le rôle du climat est incontestable dans cette histoire des fluctuations des flux hydro-sédimentaires, la part des forçages anthropiques reste à préciser au travers d'un regard dialectique, l'homme et ses aménagements ayant pu participer au contrôle des dynamiques comme y répondre par des modes d'adaptation variables suivant les époques.

\section{REMERCIEMENTS}

Les recherches ont été conduites le cadre du programme WRACC-Liger (Watershed Response to Anthropic and Climatic Change in the Ligerian high basin during Late Glacial and Holocene period; Réponse des bassins versants aux modifications climatiques et anthropiques : signatures environnementales des archives sédimentaires dans les têtes de bassin du réseau hydrographique Loire - Allier depuis le Tardiglaciaire), coordination E. Defive, avec le financement de l'Établissement Public Loire et du FEDER, projet 38207. Une partie des datations a été acquise dans le cadre de l'appel d'offre ArtémisINSHS au Centre de Datation par le RadioCarbone, UMR 5138 « Archéométrie et Archéologie », Lyon (C. Oberlin), et les mesures des teneurs en C14 à l'accélérateur ARTEMIS, UMS 2572, Laboratoire de mesure du carbone 14 (LMC14), Saclay (C. Moreau).

\section{RÉFÉRENCES BIBLIOGRAPHIQUES}

ALLEE P., 2003 - Dynamiques hydrosédimentaires actuelles et holocènes dans les systèmes fluviaux de moyenne montagne (Limousin, hautes Cévennes, Maures). Contribution à l'étude des discontinuités spatiotemporelles dans le fonctionnement du système bassin versant. Mémoire d'Habilitation à Diriger des Recherches, Université de Limoges, Limoges, 451 p.

ARCHIVES DÉPARTEMENTALES DE L'ARDÈCHE, 2006 Feuilles d'archives et forêts d'Ardèche. Catalogue d'exposition, éditions. de l'Ibie / éditions. du Chassel, 112 p.

ARNAUD F., WESSELS M., CHAPRON E., REVEL-ROLLAND M. \& DESMET M., 2008 - Signaux terrigènes dans les grands lacs subalpins: un marqueur régional à haute résolution des conditions paléohydrologiques? In GDR JURALP, Collection EDYTEM, Cahiers de Paléoenvironnement, 6, 77-88.

ARNAUD F., RÉVILLON S., DEBRET M., REVEL M., CHAPRON E., JACOB J., GIGUET-COVEXA C., POULENARD J. \& MAGNY M., 2012 - Lake Bourget regional erosion patterns reconstruction reveals Holocene NW European Alps soil evolution and paleohydrology. Quaternary Science Reviews, 51, 81-92.

ARNAUD F., POULENARD J., GIGUET-COVEX C., WILHELM B., RÉVILLON S., JENNY J.-P., REVEL M., ENTERS D. BAJARD M., FOUINAT L., DOYEN E., SIMONNEAU A., PIGNOL C., CHAPRON E., VANNIERE B. \& SABATIER P., 2016 - Erosion under climate and human pressures: An alpine lake sediment perspective. Quaternary Science Reviews, 152, 1-18.

BALLUT C., 2001 - Évolution géomorphologique et hydrologique dans les marais de Limagne au cours de la seconde moitié de l'Holocène (Massif Central, France). Quaternaire, 12 (1-2), 43-51.

BALLUT C. \& TREMENT F., 2006 - Environmental changes and evolution of settlement in the Limagne of Clermont-Ferrand (Massif Central, France). Hrvatski Geografski Glasnik, 68 (2), 5-18.

BALLUT C., PRAT B., LOPEZ SAEZ J.A., GABY G. \& CABANIS M., 2008 - Évolution environnementale d'une zone humide et de son bassin versant depuis la fin de l'âge du Fer : le maar de Montchâtre (Massif central, France). Quaternaire, 19 (1), 87-97.

BEAULIEU J.-L. de, PONS A. \& REILLE M., 1984 - Recherches pollenanalytiques sur l'histoire de la végétation des monts du Velay (Massif central, France). Dissertationes Botanicae (Festschrift Welten), 72, 45-70.

BENITO G., MACKLIN M.G., ZIELHOFER C., JONES A.F. \& MACHADO M.J., 2015 - Holocene flooding and climate change in the Mediterranean. Catena, 130, 13-33.

BERGER J.-F., 2006 - Apports récents de la paléopédologie à la définition des phases de stabilité des pédopaysages holocènes nord méditerranéens. In L. Lespez \& P. Allee (eds.), L'érosion entre société, climat et paléoenvironnement. Table ronde en l'honneur du Professeur René Neboit-Guilhot. Presses de 1'Université Blaise Pascal, Collection « Nature et Sociétés », Clermont-Ferrand, 47-62.

BERGER J.-F., 2015 - Approche socio-environnementale du Néolithique à l'Anthropocène : quelques études intégrées de la vallée du Rhône aux milieux nord méditerraneens. Mémoire d'Habilitation à Diriger des Recherches, Université Lyon 2, Lyon, 780 p.

BERGER J.-F. \& BRAVARD J.-P., 2012 - Le développement économique romain face aux crises environnementales. Le cas de la Gaule Narbonnaise. In J.-F. Berger (ed.), Des climats et des hommes. La Découverte-Inrap, Paris, 269-289.

BERGER J.-F., SALVADOR P.-G., FRANC O., VÉROT-BOURRELY A. \& BRAVARD J.-P., 2008 - La chronologie fluviale postglaciaire du haut bassin rhodanien. Collection EDYTEM, Cahiers de Paléoenvironnement, 6, 117-144.

BLANC E., 2000 - Bonnefoy, une chartreuse en Vivarais, 1176-1300. Mémoire de Maitrise d'histoire médiévale, Université Lumière Lyon II, Lyon, 320 p..

BOUVARD E., 2009 - Topographie et hydraulique des monastères cisterciens de Haute-Loire : premiers résultats et perspectives, l'exemple de l'abbaye de Bellecombe, Bulletin du Groupement de Recherches Archéologiques du Velay (GRAV), 29-38.

BOUVARD E., 2011 - L'aménagement hydraulique des sites cisterciens d'Auvergne et du Velay : des procédés vernaculaires audacieux, in Archéopages, 32, Paris, INRAP, 62-71.

BOUVARD E., 2013 - Les gestion des ressources hydrologiques des monastères d'Auvergne et du Velay, in Court, Ladet (coord.) : Les monastères de la Montagne et leur impact sur la vie sociale, économique, politique et culturelle, in revue de l'association Mémoire d'Ardèche et Temps Présent, Privas, 91-102.

BOUVARD E., 2016 - Empreintes monastiques en moyenne montagne du XIr siècle à l'Actuel. Archéologie des espaces et des paysages cisterciens dans les anciens diocèses de Clermont et du Puy. Thèse de Doctorat d'archéologie médiévale, Université Lumière Lyon 2, Lyon, 732 p. + Atlas. 
BRAVARD J.-P., 2010 - Discontinuities in braided patterns: The River Rhône from Geneva to the Camargue delta before river training. Geomorphology, 117 (3), 219-233.

BRAVARD J.-P., VEROT-BOURRELY A. \& SALVADOR P.-G., 1992 - Le climat d'après les informations fournies par les enregistrements sédimentaires fluviatiles étudiés sur des sites archéologiques. Nouvelles de l'archéologie, 50, 7-13.

BRAVARD J.-P., VEROT-BOURELY A., FRANC O. \& ARLAUD C. 1997 - Paléodynamique du site fluvial de Lyon depuis le Tardiglaciaire. In J.-P. Bravard \& D. Prestreau (eds.), Dynamique du paysage. Entretiens de géoarchéologie, documents d'Archéologie en Rhône-Alpes. Lyon, 177-201.

BRECHON F., 1998 - Contribution à une histoire de la transhumance sur le rebord Sud-Est du Massif Central : les troupeaux de l'abbaye du Monastier Saint-Chaffre à la fin du Moyen Age. In M. Framond (de) et al. (eds.), Les bénédictins de Saint-Chaffre du Monastier, histoire et archéologie d'une congrégation, Actes du colloque des 7, 8 et 9 novembre 1997, Le Monastier-sur-Gazeille, 49-65.

BRECHON F., 2000 - Réseau routier et organisation de l'espace en Vivarais et sur ses marges au Moyen Âge. Thèse de Doctorat d'Histoire, Université Lumière Lyon 2, Lyon, 4 volumes, $1362 \mathrm{p}$.

BRECHON F., 2001 - L'élevage bovin sur le plateau vivaro-vellave au Moyen Âge : aux origines du Fin Gras? Les Cahiers du Mézenc, 13, 57-66.

BÜNTGEN U., TEGEL W., NICOLUSSI K., MCCORMICK M., FRANK D., TROUET V., KAPLAN J.O., HERZIG F., HEUSSNER K.-U., WANNER H., LUTERBACHER J. \& ESPER J., 2011 - 2500 years of European climate variability and human susceptibility. Science, 331 (6017), 578-582.

CAROZZa J.-M., CAROZZa L., VALETTE P., LlUBES M., DAVID M., PY-SARAGALIA V., FERDINAND L. \& SÉVĖGNES L., 2015 - Le gisement de mégafossiles ligneux de la Bourret (moyenne vallée de la Garonne): processus de formation et signification climatique. Quaternaire. Quaternaire, 26 (4), 325-335.

CASTANET C., 2008 - La Loire en val d'Orléans. Dynamiques fluviales et socio- environnementales durant les derniers 30000 ans de l'hydrosystème à l'anthroposystème. Thèse de Doctorat, Université Paris 1 Panthéon-Sorbonne, Paris, $485 \mathrm{p}$

CHAPRON E., DESMET M., DE PUTTER T., LOUTRE M.-F., BECK C. \& DECONINCK J.-F., 2002 - Climatic variability in the northwestern Alps, France, as evidenced by 600 years of terrigenous sedimentation in Lake Le Bourget. The Holocene, 12 (2), 177-185.

CORNU L., 1991 - Economie et vie rurales sur le Plateau du Vivarais aux $X I V^{e}$ et $X V^{e}$ siècles. Mémoire de maîtrise, Université Lumière Lyon 2, Lyon, $200 \mathrm{p}$.

COUTEAUX M., 1984 - Bilan des recherches pollenanalytiques en Ardèche (France). Bulletin de la société royale de botanique de Belgique, 117 (1), Bruxelles, 181-217.

CUBIZOLLE H., 2005 - Paléoenvironnements et turfigenèse. L'apport de l'étude des tourbières à la connaissance de l'évolution holocène des milieux montagnards du Massif central oriental granitique. Mémoire d'Habilitation à Diriger des Recherches, Université de Limoges, Limoges, $750 \mathrm{p}$

CUBIZOLLE H., BONNEL P., OBERLIN C., TOURMAN A. \& PORTERET J., 2007 - Advantages and limits of radiocarbon dating applied to peat inception during the end of the Late Glacial and the Holocene: the example of mires in the Eastern Massif Central (France). Quaternaire, 18 (2), 187-208.

CUBIZOLLE H., FASSION F., ARGANT J., LATOUR C., GALET

P. \& OBERLIN C., 2012 - Mire initiation, climate change and agricultural expansion over the course of the Late-Holocene in the Massif Central mountain ranges (France): what are the causal links and what are the implications for mire conservation? Quaternary International, 251, 77-96.

CUBIZOLLE $H$, LATOUR-ARGANT $C$, DELACHANAL $S$, ARGANT J., DENDIEVEL A.-M., DIETRE B., THEBAUD G. \& GALER P., 2016 - Développement de l'emprise agro-pastorale et changements hydro-pédologiques à l'Holocène récent : l'exemple de la vallée du ruisseau des barges dans le Massif Central oriental (France), Quaternaire, 27 (2), 91-109.

DANSGAARD W., JOHNSEN S.J., MOLLER J. \& LANGWAY J.C.C., 1969 - One thousand centuries of Climatic Record from Camp Century in the Greenland Ice sheet. Science, 166 (3903), 377-380.

DEBRET M., CHAPRON E., DESMET M., ROLLAND-REVEL M., MAGAND O.-M., TRENTESAUX A., BOUT-ROUMAZEILLE V., NOMADE J. \& ARNAUD F., 2010 - North western Alps Holocene paleohydrology recorded by flooding activity in Lake Le Bourget, France and its relationship with Mont-Blanc glacier fluctuations. Quaternary Science Review 29 (17-18), 2185-2200.

DEFIVE E., 1996 - L'encaissement du réseau hydrographique dans le bassin supérieur de la Loire. Contribution à l'étude des rythmes d'évo- lution géomorphologique en moyenne montagne volcanisée. Thèse de Doctorat, Université Paris I Panthéon-Sorbonne, Paris, $577 \mathrm{p}$.

DEFIVE E., 2010 - L'évolution post-glaciaire et holocène des versants sur les hauts plateaux du Velay oriental : facteurs naturels et rôle de l'anthropisation, première approche. In D. RICARD (ed.) Développement durable des territoires : de la mobilisation des acteurs aux démarches participatives. Sixième colloque franco-polonais: Presses Universitaires Blaise Pascal et CERAMAC, 28, 395-410.

DEFIVE E., 2013 - Réponse hydro-sédimentaire des têtes de bassin aux fluctuations environnementales historiques en contexte de moyenne montagne tempérée : l'exemple du Velay (Sud-Est du Massif central, France), premiers résultats. Quaternaire, 24 (4), 461-476

DEFIVE E. \& POIRAUD A., 2013 - Velay and Mézenc Massif: harsh volcanic highlands around the Loire River source. In M. Fort \& M.-F. André (eds.), Landscapes and Landforms of France. Springer Verlag, 93-103.

DEFIVE E., COURRIOUX G., PROGNON C. \& POIRAUD A., 2011 - Carte géologique de la France à 1/50 000 ( $\mathrm{n}^{\circ}$ 816), Le Monastier-sur-Gazeille (XXVIII-36). Carte et notice explicative. Bureau de Recherches Géologiques et Minières, Orléans, 196 p.

DEFIVE E, ADAMIEC G., BARRA A., BEAUGER A, BERGER J.-F., BOUVARD E., BRAUCHER R., CABANIS M., DELVIGNE V., DENDIEVEL A.-M., GUILLOU H., GUNNELL Y., LAFARGE A., MIRAS Y., NOMADE S., POIRAUD A., RAYNAL J.-P.,VIRMOUX C. \& VOLDOIRE O., 2015 - Réponse des bassins versants aux modifications climatiques et anthropiques: signatures environnementales des archives sédimentaires dans les têtes de bassin du réseau hydrographique Loire - Allier depuis le Tardiglaciaire. Rapport scientifique final 2015, Etablissement Public Loire, Projet 38207, vol. 1, 191 p., vol. 2, 337 p. doi: $10.13140 /$ RG.2.1.1840.7124.

DELILE H., SCHMITT L., JACOB-ROUSSEAU N., GROSPRETRE L., PRIVOLT G. \& PREUSSER F., 2016 - Headwater valley response to climate and land use changes during the Little Ice Age in the Massif Central (Yzeron basin, France). Geomorphology, 257, 179-197.

DENDIEVEL A.-M., 2012 - Définition du potentiel paléo-environnemental et géo-archéologique du Massif du Mézenc (Massif Central, France). Mémoire de Master 1, Université Jean Monnet, Saint-Etienne, $70 \mathrm{p}$.

DENDIEVEL A.-M., HAAS J.-N., DELRIEU F, \& CUBIZOLLE H., 2014 - Nouvelles données paléoenvironnementales et archéologiques sur le plateau du Béage, La tourbière de La Narce et ses environs, premiers résultats. Ardèche Archéologie, 31, 27-33.

DENDIEVEL A.-M., CUBIZOLLE H., DIETRE B., GAERTNER V, GALLICE F, JOUANNIC G. \& HAAS J.-N, 2015 - Changements environnementaux du Tardiglaciaire à l'Holocène moyen dans le Velay oriental : la séquence sédimentaire de La Narce du Béage en Ardèche (Massif Central, France). Quaternaire, 26 (4), 263-276.

DENDIEVEL A.-M., ARGANT J., CUBIZOLLE H., DELRIEU F., DENG-AMIOT Y., JACCOTTEY L., MILLE P. \& KURZAJ M.-C., 2016 - Le plateau du Béage au second âge du Fer (Ardèche) : le site du "Crouzet - La Veysse » et son environnement. Bulletin de l'AFEAF, 34, 39-42.

EVIN J., FORTIN P. \& OBERLIN C. 1995 - Calibration et modes de représentation des datations radiocarbone concernant le Néolithique de l'Est et du Sud-Est de la France. In J.-L. Voruz (ed.), Chronologies néolithiques de 6000 à 2000 ans avant notre ère dans le Bassin Rhodanien, Colloque d'Ambérieu-en-Bugey, 19-20 sept.1992, Société Préhistorique Rhodanienne, Doc. du département d'anthropologie et d'écologie de l'Université de Genève, 20, 31-39.

FEL A. 1962 - Les Hautes terres du Massif central, tradition paysanne et économie agricole. PUF, Paris, $340 \mathrm{p}$.

FOURNIER C., 2014 - Occupation du sol, aménagements et pratiques (du XVIII siècle à nos jours) : apports à l'étude du fonctionnement hydro-sédimentaire du bassin versant de Champetienne (43). Mémoire de Master 1, Université Blaise Pascal, Clermont-Ferrand 2, ClermontFerrand, $57 \mathrm{p}$

GOB F., 2005 - La lichenométrie appliquée à l'étude de rivières en gorge en milieu méditerranéen : caractérisation de leurs paramètres dynamiques et de leur évolution géomorphologique durant le Petit Âge Glaciaire. Thèse de Doctorat, Université de Liège, Liège, $307 \mathrm{p}$.

GOB F., JACOB N., BRAVARD J.-P. \& PETIT F., 2008 - The value of lichenometry and historical archives in assessing the incision of submediterranean rivers from the Little Ice Age in the Ardèche and upper Loire (France). Geomorphology, 94 (1-2), 170-183.

GROVE J.M., 2001 - The initiation of the "Little Ice Age" in regions round the North Atlantic. J. Climatic Change, 48 (1), 53-82.

GIGUET-COVEX C., ARNAUD F., POULENARD J., DISNAR J.-R., DELHON C., FRANCUS P., DAVID F., ENTERS D., REY P.-J. \& DELANNOY J.-J., 2011 - Changes in erosion patterns during the Holocene in a currently treeless subalpine catchment inferred from lake sediment geochemistry (Lake Anterne, $2063 \mathrm{~m}$ asl, NW French 
Alps): the role of climate and human activities. The Holocene, 21 (4), 651-664.

HOFFMANN T., LANG A. \& DIKAU R., 2008a - Holocene river activity: analysing 14C-dated fluvial and colluvial sediments from Germany. Quaternary Science Reviews, 27 (21), 2031-2040.

HOFFMANN T., ERKENS G., GERLACH R., KLOSTERMANN J. \& LANG A. 2008b - Trends and controls of Holocene floodplain sedimentation in the Rhine catchment. Catena, 77 (2), 93-106.

HOLZHAUSER H., 1997 - Fluctuations of the Grosser Aletsch Glacier and the Gorner Glacier during the last 3200 years: new results. In B. Frenzel (ed.), Glacier fluctuations during the Holocene: Akademie der Wissenschaften und der Literatur, Paläoklimaforschung/Palaeoclimate Research, 24, 35-58.

HOLZHAUSER H., MAGNY M. \& ZUMBUÜHL H.J., 2005 - Glacier and lake-level variations in west-central Europe over the last 3500 years. The Holocene, 15 (6), 789-801.

IVY-OCHS S., KERSCHNER H., MAISCH M., CHRISTL M., KUBIK P.W. \& SCHLÜCHTER C., 2009 - Latest Pleistocene and Holocene glacier variations in the European Alps. Quaternary Science Reviews, 28 (21), 2137-2149.

JACOB N. \& ASTRADE L., 2014 - Phénomènes torrentiels et transferts sédimentaires autour du massif du Tanargue, de l'âge du fer au petit âge de glace (PAG) (Cévennes vivaraises, France). Revue Géographique des Pays Méditerranéens, 122, 81-97.

JACOB N., ASTRADE L. \& BRAVARD J.-P., 2006 - Le comportement hydrosédimentaire de rivières torrentielles des Cévennes et des Préalpes au Petit Âge Glaciaire. Enseignements géomorphologiques des archives historiques, de la dendrochronologie et de la lichénométrie. In P. Allée \& L. Lespez (eds.), L'érosion entre société, climat et paléoenvironnement, Presses universitaires Blaise Pascal, ClermontFerrand, 297-310.

JOHNSTONE E., MACKLIN M.G. \& LEWIN, J., 2006 - The development and application of a database of radiocarbon-dated Holocene fluvial deposits in Great Britain. Catena, 66 (1), 14-23.

LARUE J.-P., 2009 - Etude morphologique et dynamique des têtes de vallon en milieux forestiers et cultivés : exemple dans le nord du Massif central francais. Norois, 212 (3), 53-68.

LÉOGIER J.-J., 1995 - La conquête du Mézenc illustrée. Les Cahiers du Mézenc, 7, 45-53.

LÉOGIER J.-J. 2005 - Contribution à l'histoire de l'agriculture du massif du Mézenc. Une nouvelle approche du compoix des Estables de 1550. Les Cahiers du Mézenc, 17, 33-40.

LE ROY M., NICOLUSSI K., DELINE P., ASTRADE L., EDOUARD J.-L., MIRAMONT C. \& ARNAUD F., 2015 - Calendardated glacier variations in the western European Alps during the Neoglacial: the Mer de Glace record, Mont Blanc massif. Quaternary Science Reviews, 108, 1-22.

LE ROY LADURIE E., 2004 - Histoire humaine et comparée du climat, volume 1 : Canicules et glaciers (XIII ${ }^{e}-X V I I I^{e}$ siècles). Fayard, $740 \mathrm{p}$.

LE ROY LADURIE E., ROUSSEAU D. \& VASAK A., 2011 - Les fluctuations du climat de l'an mil à aujourd'hui. Editions FAYARD, Paris, $321 \mathrm{p}$.

LESPEZ L. (éd.), 2012 - Paysages et gestion de l'eau. Sept millénaires d'histoire de vallées et de plaines littorales en Basse-Normandie. Co-édition Presses universitaires de Caen / MRSH, $n^{\circ}$ hors série des Enquêtes Rurales, 3, 336 p.

L'HÉRITIER N., 2012 - Les têtes de bassin : de la cartographie aux échelles mondiale et française à la caractérisation des ruisseaux limousins. Thèse de Doctorat, Université de Limoges, Limoges, $481 \mathrm{p}$.

LUGAN M., 2014 - Inventaire des anciens aménagements hydrauliques sur le ruisseau de Mazan : la part des héritages anthropique dans l'évolution paysagère d'un cours d'eau du haut plateau ardéchois. Mémoire de Master 1, Université Jean Moulin, Lyon 3, Lyon, 82 p.

MACKLIN M.G. \& LEWIN J., 2003 - River sediments, great floods and centennial-scale Holocene climatic change. Journal of Quaternary Science, 18 (2), 101-105.

MACKLIN M.G., JONES A.F. \& LEWIN J., 2010 - River response to rapid Holocene environmental change: evidence and explanation in British catchments. Quaternary Science Reviews, 29 (13), 1555-1576. MAGNY M., 2004 - Holocene climatic variability as reflected by midEuropean lake-level fluctuations, and its probable impact on prehistoric human settlements. Quaternary International, 113 (1), 65-79.

MAGNY M., ARNAUD F., HOLZHAUSER H., CHAPRON E., DEBRET M., LEROUXA., MILLET L., REVEL M. \& VANNIERE B., 2010 - Solar and site-sensitivity imprints on palaeohydrological records for the last millennium in west-central Europe. Quaternary Research, 73 (2), 173-179.

MANEN C. \& SABATIER P., 2003 - Chronique radiocarbone de la Néolithisation en Méditerranée nord-occidentale. Bulletin de la Société Préhistorique de France, 100 (3), 479-504.
MERGOIL J. \& BOIVIN P., 1993 - Le Velay, son volcanisme et les formations associées. Notice de la carte à $1 / 100000$. Géologie de la France, 3, 96 p., carte hors texte.

MICHCZYNSKA D.J. \& PAZDUR A., 2004 - Shape analysis of cumulative probability density function of radiocarbon dates set in the study of climate change in the Late Glacial and Holocene. Radiocarbon, 46 (2), 733-744.

MICHCZYNSKA D.J., MICHCZYNSKI A. \& PAZDUR, A., 2007 - Frequency distribution of radiocarbon dates as a tool for reconstructing environmental changes. Radiocarbon, 49 (2), 799-806.

MIRAMONT C. \& GUILBERT X., 1997 - Variations historiques de la fréquence des crues et évolution de la morphogenèse fluviale en moyenne Durance (France du Sud-Est) / Historical changes of floods frequency and fluvial morphogenesis in the middle Durance fluvial system (South-eastern France). Géomorphologie : relief, processus, environnement, 3 (4), 325-337.

MIRAS Y., SURMELY F., GUENET P., VANNIÈRE B., WALTERSIMONNET A.V. \& TZORTZIS S., 2006 - Dynamiques d'occupation et histoire de l'environnement d'un terroir de moyenne montagne : la tourbière de Peyre (Lacapelle-Barrès, Cantal, Massif central) et ss alentours. Premiers résultats. In Y. Miras Y. et F. Surmely (eds.), Gestion des Paléoenvironnements et stratégies d'exploitation des milieux en moyenne montagne par les sociétés humaines, Actes de la table ronde de Pierrefort, juin 2003, Collection des Annales Littéraires, Série « Environnement, sociétés et archéologie », $\mathrm{n}^{\circ}$ 9, Presses Universitaires de Franche-Comté, Besançon, 157-182.

MIRAS Y., BEAUGER A., LAVRIEUX M., BERTHON V., SERIEYSSOL K., ANDRIEU-PONEL V. \& LEDGER P. 2014 - Tracking long-term human impacts on landscape, vegetal biodiversity and water quality in the lake Aydat (Auvergne, France) using pollen, non-pollen palynomorphs and diatom assemblages. Palaeogeography, Palaeohydrology, Palaeoecology, 424, 76-90.

MOLINIER A., 1985 - Stagnations et croissance : le Vivarais aux $X V I I^{e}$ et XVIII siècles. Paris, Éditions de l'EHESS / Jean Touzot, libraireéditeur, $500 \mathrm{p}$.

NOTEBAERT B. \& BERGER J.-F., 2014 - Quantifying the anthropogenic forcing on soil erosion during the Iron Age and Roman Period in southeastern France. Anthropocene, 8, 59-69.

NOTEBAERT B., BERGER J.-F. \& BROCHIER J.-L. 2014 - Characterization and quantification of Holocene colluvial and alluvial sediments in the Valdaine Region (southern France). The Holocene, 24 (10), 1-16.

NUSSBAUMER S.U., STEINHILBER F., TRACHSEL M. BREITENMOSER P., BEER J., BLASS A., GROSJEAN M. HAFNER A., HOLZHAUSER H., WANNER H. \& ZUMBÜHL H.J., 2011 - Alpine climate during the Holocene: a comparison between records of glaciers, lake sediments and solar activity. Journal of Quaternary Science, 26 (7), 703-713.

RÉGNÉ J., 1923 - La Vie monastique en Vivarais au moyen âge. La Chartreuse de Bonnefoy. Collection extrait de la « revue Mabillon », E. Aubin, Ligugé (Vienne), $40 \mathrm{p}$.

REUMAUX E., 2002 - La mise en place d'une forêt domaniale au mont Mézenc (1860-1914), point culminant d'Ardèche et de HauteLoire. Mémoire de Maîtrise d'histoire contemporaine, Université Grenoble 2, Grenoble, 201 p.

REUMAUX E., 2003 - Une forêt et des hommes : l'entreprise de reboisement au Mézenc. Les Cahiers du Mézenc, 15, 31-46

RHOUJJATI A., 1995 - Géochimie organique et changements environnementaux du dernier cycle climatique : étude des sédiments du lac de Saint Front (Massif Central, France). Thèse de Doctorat en Géologie du Quaternaire, Université Aix-Marseille 2, 194 p.

SALVADOR P.-G. \& BERGER J.-F., 2014 - The evolution of the Rhone River in the Basses Terres basin during the Holocene (Alpine foothills, France). Geomorphology, 204, 71-85.

SCHMITT L, VALETTE L, VALIN K, PIEGAY H, \& HALLOT E., 2005 - Proposition d'une méthode de typologie hydro-géomorphologique des cours d'eau et test sur un sous-bassin du Rhône (bassin de 1'Yzeron). Mosella, 29 (3-4), 323-340.

SCHÖNWIESE C., 1995 - Klimaänderungen: Daten, Analysen, Prognosen. Springer, Heidelberg, $244 \mathrm{p}$

SHENNAN S. \& EDINBOROUGH K., 2007 - Prehistoric population history: from the Late Glacial to the Late Neolithic in Central and Northern Europe. Journal of Archaeological Science, 34 (8), 1339-1345.

SIMANDOUX J., 2007 - Dynamique contemporaine des paysages végétaux dans le massif du Mézenc (Massif central). Apport d'une analyse diachronique à l'étude de la reconquête arborée des versants. Mémoire de Master 1, Université Blaise-Pascal Clermont-Ferrand 2, Clermont-Ferrant, $111 \mathrm{p}$.

SIMANDOUX J., 2008 - Dynamique contemporaine des paysages végétaux dans le massif du Mézenc (Massif central). Caractérisa- 
tion des modalités et des vitesses de colonisation des ligneux hauts. Mémoire de Master 2, Université Blaise Pascal Clermont-Ferrand 2, $94 \mathrm{p}$.

STARKEL L., SOJA R. \& MICHCZYNSKA D.J., 2006 - Past hydrological events reflected in Holocene history of Polish rivers. Catena, $\mathbf{6 6}$ (1), 24-33.

STRAHLER A.N., 1957 - Quantitative analysis of watershed geomorphology. Transactions of the American Geophysical Union, 38 (6), 913-920.

TREMENT F., DELPY M., FASSION F. \& MASSOUNIE G., 2014 - Centres et périphéries dans les cités antiques du Massif Central. Occupation, mise en valeur et intégration des territoires de montagne dans la cité des Arvernes (fin de l'âge du Fer - début du Moyen Âge). In Franges urbaines et confins territoriaux, la Gaule dans l'Empire. Actes du Colloque, Calenda, 589-614.

VALADAS B., 1984 - Les hautes terres du Massif central français. Contribution à l'étude des morphodynamiques récentes sur versants cristallins et volcaniques. Thèse de Doctorat d'Etat, Université Paris I Panthéon-Sorbonne, 2 tomes, Paris, 927 p.
WILHELM B., ARNAUD F., SABATIER P., CROUZET C., BRISSET E., CHAUMILLON E., DISNAR J.-R., GUITER F., MALET E., REYSS J.-L., TACHIKAWA K., BARD E. \& DELANNOY J.-J., 2012 - 1400 years of extreme precipitation patterns over the Mediterranean French Alps and possible forcing mechanisms. Quaternary Research, 78 (1), 1-12.

WILLIAMS A.N., 2012 - The use of summed radiocarbon probability distributions in archaeology: a review of methods. Journal of Archaeological Science, 39 (3), 578-589.

ZIELHOFER C. \& FAUST D., 2008 - Mid-and Late Holocene fluvial chronology of Tunisia. Quaternary Science Reviews, 27 (5), 580-588. ZIELHOFER C., BUSSMANN J., IBOUHOUTEN H. \& FENECH K., 2010 - Flood frequencies reveal Holocene rapid climate changes (Lower Moulouya River, northeastern Morocco). Journal of Quaternary Science, 25 (5), 700-714.

ZUMBÜHL H.J. \& HOLZHAUSER H., 1988 - Glaciers des Alpes $d u$ Petit âge glaciaire. Editions du Club Alpin Suisse, Berne, 322 p. 